\title{
Inhibiting neutral amino acid transport for the treatment of phenylketonuria
}

\author{
Adam M. Belanger, Malgorzata Przybylska, Estelle Gefteas, Matthew Furgerson, Sarah Geller, \\ Alla Kloss, Seng H. Cheng, Yunxiang Zhu, and Nelson S. Yew \\ Sanofi, Framingham, Massachusetts, USA.
}

\begin{abstract}
The neuropathological effects of phenylketonuria (PKU) stem from the inability of the body to metabolize excess phenylalanine (Phe), resulting in accumulation of Phe in the blood and brain. Since the kidney normally reabsorbs circulating amino acids with high efficiency, we hypothesized that preventing the renal uptake of Phe might provide a disposal pathway that could lower systemic Phe levels. SLC6A19 is a neutral amino acid transporter responsible for absorption of the majority of free Phe in the small intestine and reuptake of Phe by renal proximal tubule cells. Transgenic KO mice lacking SLC6A19 have elevated levels of Phe and other amino acids in their urine but are otherwise healthy. Here, we crossed the Pah enu2 mouse model of PKU with the SIc6a19-KO mouse. These mutant/KO mice exhibited abundant excretion of Phe in the urine and an approximately $70 \%$ decrease in plasma Phe levels. Importantly, brain Phe levels were decreased by $\mathbf{5 0} \%$, and the levels of key neurotransmitters were increased in the mutant/KO mice. In addition, a deficit in spatial working memory and markers of neuropathology were corrected. Finally, treatment of Pahnu2 mice with SIc6a19 antisense oligonucleotides lowered Phe levels. The results suggest that inhibition of SLC6A19 may represent a novel approach for the treatment of PKU and related aminoacidopathies.
\end{abstract}

\section{Introduction}

As with all the other amino acids, free phenylalanine (Phe) is carefully regulated to maintain steady-state levels in the body. Inputs of Phe come mainly from the enzymatic digestion of dietary proteins and the normal turnover of proteins in tissues, while outputs of free Phe include hydroxylation to tyrosine, incorporation into new proteins, and, to a minor extent, transamination to phenylketones that are excreted in the urine $(1,2)$. The primary catabolic pathway for Phe is its conversion to tyrosine by Phe hydroxylase (PAH) (3). In patients with the autosomal recessive disorder phenylketonuria (PKU) this pathway is disrupted or impaired due to mutations or deletions in the gene encoding PAH (4). Very rarely PAH activity is diminished due to defects in the synthesis or regeneration of tetrahydrobiopterin, the essential cofactor for the enzyme (5). In either case, the consequence is an accumulation of Phe in blood and tissues. Although the pathological mechanism is not completely understood, elevated Phe levels have been reported to underlie several neuropathological alterations in the brain, including reduced myelination, impaired dendritic arborization, gliosis, oxidative stress, decreased levels of neurotransmitters (dopamine, serotonin, norepinephrine), and decreased cerebral protein synthesis and glucose metabolism (6-8). If untreated, PKU leads to severe and irreversible mental retardation. Adherence to a strict low-Phe diet initiated soon after birth prevents most of the severe manifestations of the disease. However, compliance is often poor, and the majority of adolescents and adults have Phe levels above the recommended range, resulting in poor academic performance and emotional, behavioral, and social problems $(9,10)$. In addition, even stricter control of Phe intake is of crucial importance for women who become pregnant, since elevated Phe levels are highly teratogenic to the developing fetus (11). This regimen is very difficult, and, consequently, the frequency of newborns with neurocognitive defects remains unacceptably high (12). Therefore, there is a significant unmet medical need for therapies that can at least partially restore Phe homeostasis and bring free Phe toward more normal levels.

The highly efficient retrieval of filtered peptides and amino acids by the kidney acts as a barrier against removal of excess Phe from the blood. Reabsorption of nearly all amino acids in the glomerular filtrate occurs in the proximal convoluted tubule, whose cells contain transporters for cationic, anionic, and neutral amino acids $(13,14)$. Solute carrier family 6 member 19 (SLC6A19) is a sodium/neutral amino acid symporter that is localized to the apical membrane of the proximal tubule (15). SLC6A19 has a preference for 


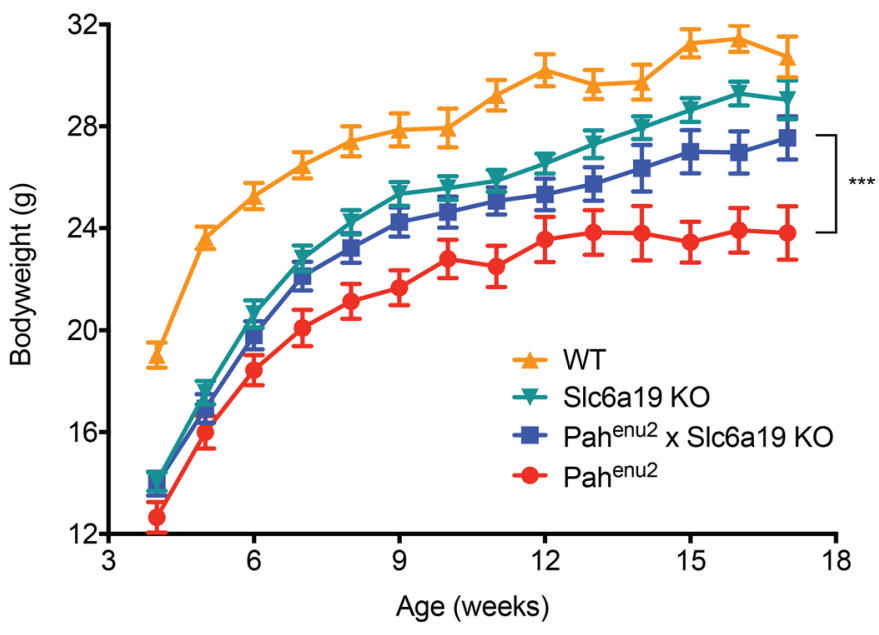

Figure 1. Growth curves of the different genotypes of mice. $\mathrm{KO}$, homozygous KO. $n=7-34$ animals (males)/ genotype. One-way ANOVA was performed, follow by Tukey's multiple comparisons test. ${ }^{* *} P<0.0001$, weeks $15-17$. Mean \pm SEM is shown.

large- and branched-chain neutral amino acids, such as leucine, isoleucine, methionine, and glutamine, but transports all neutral amino acids, including Phe, tyrosine, and tryptophan. The associated transmembrane protein 27 (TMEM27), also known as collectrin, is required for the proper trafficking and localization of SLC6A19 to the apical membrane (16-18). In addition to SLC6A19's role in the kidney, the transporter is also expressed in the enterocytes of the small intestine, where it is responsible for absorption of the majority of free neutral amino acids. The obligate partner for intestinal SLC6A19, where TMEM27 is absent, is angiotensin-converting enzyme 2 (ACE2), whose C-terminus is homologous to TMEM27 (19). Mice lacking SLC6A19 exhibit neutral aminoaciduria, reduced body weight, and a decreased insulin response but have a normal life span and are otherwise healthy (20).

In this study, we investigated the effects of blocking neutral amino acid transport in the $P a h^{\text {enu2 }}$ mouse model of PKU. Pah ${ }^{\text {enu2 }}$ mice contain a point mutation in the active site of the PAH enzyme that abolishes enzyme activity, resulting in Phe levels that are 10- to 20-fold higher than normal (21). Furthermore, $P a h^{\text {enu2 }}$ mice share several features with untreated human PKU patients, including retarded growth, microcephaly, deficiencies in neurogenic amines, and behavioral and memory deficits (22-25). We utilized two approaches to inhibit neutral amino acid transport. First, we generated $P a h^{\text {enu2 }}$ mice lacking SLC6A19. Second, we used antisense oligonucleotides to knock down SLC6A19 in the kidney of the Pah ${ }^{\text {enu2 }}$ mouse. Both approaches resulted in substantial excretion of Phe in the urine and marked improvements in the biochemical and neurologic abnormalities present in the $P a h^{\text {enu2 }}$ mouse.

\section{Results}

Generation of Pah ${ }^{\text {enu2 }}$ mice lacking SLC6A19. To determine the effect of completely abolishing SLC6A19 function in the $P a h^{\text {enu2 }}$ mouse, heterozygous $P a h^{\text {enu2 }}$ females were mated with heterozygous Slc6a19-KO (Slc6a19-KO) males to generate mice heterozygous for both the Pah ${ }^{\text {enu2 }}$ and Slc6a19 genes. Pah ${ }^{\text {enu2 } / S l c 6 a 19}$ heterozygous mice were then intercrossed to generate homozygous Pah ${ }^{\text {enu } 2}$ mice lacking Slc6a19. We confirmed the absence of SLC6A19 expression by probing kidney lysates with antibodies against SLC6A19 (data not shown). The Pah ${ }^{\text {enu2 }} \times$ Slc6a19-KO mice were generated at the expected Mendelian frequency, and the offspring were healthy and normal in appearance.

We monitored the growth of the different genotypes of mice through the first 4 months after birth. At weaning, wild-type mice were approximately $30 \%$ heavier than the Pah ${ }^{\text {enu } 2}$, Slc6a19, and Pah ${ }^{\text {enu } 2} \times$ Slc6a19-KO mice, and they maintained this elevated weight throughout the first 16 weeks (Figure 1). The Pahn ${ }^{\text {en } 2}$ mice were the smallest of the 4 genotypes, with their weight appearing to plateau beginning at approximately 12 weeks of age. The Slc6a19-KO mice were also small at weaning but continued to gain weight through week 16. The growth of the Pah ${ }^{\text {enu2 }} \times$ Slc6a19-KO mice roughly paralleled that of the Slc6a19-KO animals, with their growth rate falling midway between that of the wild-type and $P a h^{\text {enu2 }}$ mice (Figure 1). The results demonstrate that the absence of SLC6A19 had a beneficial effect on postnatal growth in the context of the Pahu ${ }^{\text {en }}$ mutation.

Abundant excretion of Phe in the urine of Pah ${ }^{\text {enu2 }}$ mice lacking SLC6A19. To determine the degree of aminoaciduria induced by knocking out Slc6a19, mice were placed in metabolic cages and urine was collected over a 24-hour period. Wild-type mice excreted negligible amounts of amino acids in the urine, as expected (Table 1). 
Table 1. Urine amino acid levels

\begin{tabular}{|c|c|c|c|c|c|}
\hline Amino acid & $P a h^{\text {enu2 }} \times$ SIc6a19-KO urine $(\mu \mathrm{g})$ & $P a h^{\text {enu2 }} \times$ SIc6a19 Het urine $(\mu \mathrm{g})$ & $P a h^{\text {enu2 }}$ urine $(\mu \mathrm{g})$ & SIc6a19-KO urine $(\mu \mathrm{g})$ & Wild-type urine $(\mu \mathrm{g})$ \\
\hline Glycine & $3,808 \pm 1,325$ & $1,313 \pm 1,156$ & $73 \pm 30$ & $4,471 \pm 39$ & $60 \pm 22$ \\
\hline Serine & $4,404 \pm 790$ & $547 \pm 523$ & $43 \pm 22$ & $5,214 \pm 1,272$ & $30 \pm 13$ \\
\hline Asparagine & $2,064 \pm 680$ & $195 \pm 185$ & $47 \pm 16$ & $1,878 \pm 875$ & $31 \pm 3$ \\
\hline Aspartic acid & $156 \pm 106$ & $42 \pm 19$ & $32 \pm 17$ & $353 \pm 261$ & $25 \pm 10$ \\
\hline Glutamine & $47,873 \pm 8,276$ & $2,681 \pm 4,144$ & $76 \pm 36$ & $56,458 \pm 17,392$ & $118 \pm 79$ \\
\hline Glutamic acid & $884 \pm 363$ & $74 \pm 35$ & $47 \pm 17$ & $1,029 \pm 543$ & $91 \pm 67$ \\
\hline Cystine & $1,156 \pm 608$ & $128 \pm 27$ & $26 \pm 13$ & $1,199 \pm 174$ & $37 \pm 44$ \\
\hline Lysine & $1,639 \pm 1,037$ & $63 \pm 11$ & $37 \pm 9$ & $1,685 \pm 923$ & $38 \pm 11$ \\
\hline Proline & $58 \pm 18$ & $88 \pm 127$ & $27 \pm 2$ & $84 \pm 44$ & $24 \pm 8$ \\
\hline Histidine & $4,216 \pm 521$ & $877 \pm 753$ & $53 \pm 27$ & $4,565 \pm 645$ & $33 \pm 17$ \\
\hline Tyrosine & $4,373 \pm 978$ & $314 \pm 253$ & $30 \pm 11$ & $7,522 \pm 1,774$ & $29 \pm 8$ \\
\hline Phenylalanine & $13,607 \pm 779$ & $2,979 \pm 2,394$ & $256 \pm 119$ & $3,328 \pm 516$ & $27 \pm 2$ \\
\hline Tryptophan & $513 \pm 73$ & $142 \pm 111$ & $14 \pm 4$ & $523 \pm 75$ & $10 \pm 3$ \\
\hline Valine & $5,358 \pm 1630$ & $179 \pm 68$ & $28 \pm 12$ & $6,103 \pm 352$ & $21 \pm 6$ \\
\hline
\end{tabular}

Total amino acid mass (concentration $\times$ volume) after 24 -hour urine collection.

$P a h^{\text {enu2 }}$ mice likewise excreted very small amounts of amino acids with the exception of Phe, with Phe levels 10-fold higher those of wild-type mice (Table 1 and Figure 2A). This increase is likely the result of the extremely elevated Phe levels in the blood, exceeding the capacity of the renal proximal tubule to reabsorb Phe. Slc6a19-KO mice excreted substantial amounts of almost all amino acids, reflecting the broad substrate specificity of this transporter. Glutamine, the most abundant amino acid in blood, was also the most abundant amino acid present in the urine of the Slc6a19-KO animals, followed by the neutral amino acids alanine, leucine, and methionine (Table 1). Phe amounts in the urine of Slc6a19-KO mice were elevated more than 100-fold over those of wildtype mice (3,328 $\pm 516 \mu \mathrm{g}$ versus $27 \pm 2 \mu \mathrm{g}$ : total excreted over a 24 -hour period). Phe amounts in the urine of $P a h^{\text {enu } 2} \times$ heterozygous Slc6a19 mice $\left(P a h^{\text {enu } 2} \times\right.$ Slc6a19 Het mice) were also elevated, likely reflecting a combination of the partial loss of transporter activity and the highly elevated Phe levels in the Pah ${ }^{\text {enu2 }}$ mice. However, Phe levels were by far the highest in the urine of $\mathrm{Pah}^{\mathrm{n} u 2} \times$ Slc6a19-KO mice, which excreted 500-fold more Phe in the urine $(13,607 \pm 779 \mu \mathrm{g})$ compared with wild-type animals and 50-fold more Phe compared with $P a h^{\text {enu2 }}$ mice (Table 1 and Figure 2A). Thus, genetic deletion of Slc6a19 in $P_{a h} h^{\text {enu2 }}$ mice promoted impressive excretion of Phe in the urine.

Dramatic reductions in blood and brain Phe levels in Pahenu2 mice lacking SLC6A19. Because loss of SLC6A19 led to severe urinary aminoaciduria, we next assessed what effect loss of this transporter had on amino acid levels in the blood. Despite the marked accumulation of a broad spectrum of amino acids in the urine, the levels of amino acids in plasma were comparable in either mice containing or lacking Slc6a19 (Table 2). For example, tyrosine levels were similar in wild-type $(140 \pm 30 \mu \mathrm{M})$ and Slc6a19-KO $(134 \pm 10 \mu \mathrm{M})$ animals and comparably lower both in $P^{h^{\text {enu2 }}}$ mice $(68 \pm 10 \mu \mathrm{M})$ and in $P a h^{\text {enu2 }} \times$ Slc6a19-KO mice $(75 \pm 12 \mu \mathrm{M})$. The important exception was Phe, by far the most abundant amino acid $(1,875 \pm 328 \mu \mathrm{M})$ in plasma of $\mathrm{Pah}^{\text {enu2 }}$ mice, which decreased by approximately $70 \%$ (to $573 \pm 105 \mu \mathrm{M}$ ) in Pah ${ }^{\text {enu2 }} \times$ Slc6a19-KO animals (Table 2 and Figure 2B). Thus, loss of SLC6A19 in Pah ${ }^{\text {enu2 }}$ mice substantially and specifically reduced Phe relative to other circulating amino acids.

As was observed in plasma, similar levels of amino acids were present in the brains of both wild-type and Slc6a19-KO mice (Table 3). In Pahenu2 mice, brain Phe levels were elevated 5-fold relative to levels in wild-type animals (Figure 2C). In Pah $h^{\text {enu }} \times S l c 6 a 19$ Het mice, even though there was increased excretion of Phe in urine, brain Phe levels did not decrease relative to the levels in $P_{a h}{ }^{\text {en2 }}$ mice (Figure 2C). However, in $P a h^{\text {enu2 }} \times$ Slc6a19-KO mice, brain Phe levels decreased by 50\% relative to the levels in $P a h^{\text {enu2 }}$ mice. Notably, 

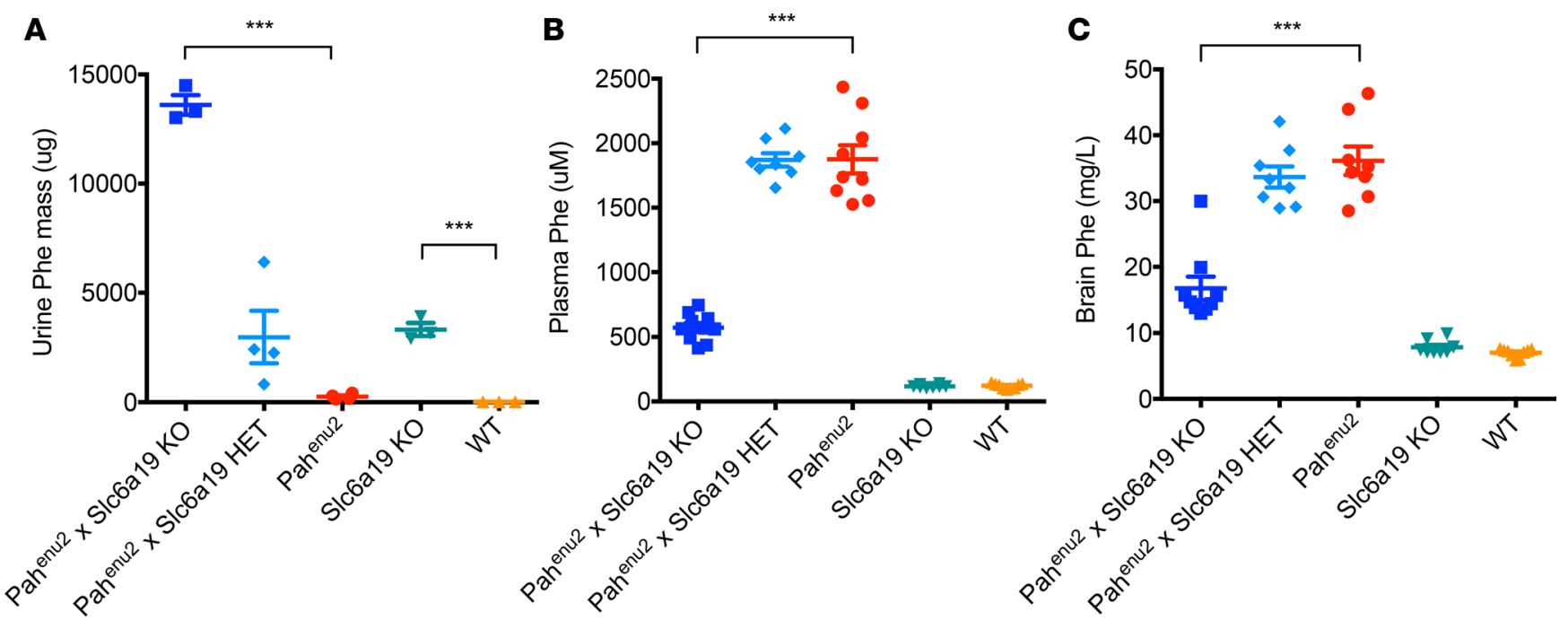

Figure 2. Decreased Phe levels in Pah ${ }^{\text {enu2 }}$ mice lacking SIc6a19. (A) Excretion of Phe in the urine of Pahenu2 $\times$ SIc6a19-KO mice. Mice were placed in metabolic cages, and urine was collected for 24 hours. (B) Phe levels in plasma. (C) Phe levels in the brain. Animals were perfused with PBS and whole brains were harvested. Phe was quantitated by HPLC/tandem MS/MS. Phe is expressed as milligram Phe per liter of brain homogenate. $n=8-10$ animals/group. One-way ANOVA was performed, follow by Tukey's multiple comparisons test. ${ }^{* *} P<0.0001$. Mean $\pm \mathrm{SEM}$ is shown.

brain tyrosine and tryptophan levels in $P a h^{\text {enu2 }} \times$ Slc6a19-KO mice were unchanged relative to the levels in wild-type animals (Table 3). These results demonstrate that deletion of Slc6a19 resulted in strikingly significant decreases in both plasma and brain Phe levels, with modest effects on the levels of other amino acids.

Restoration of multiple neurogenic amines in the brains of Pahnu2 mice lacking SLC6A19. Prior to the widespread implementation of newborn screening and dietary Phe restriction, early studies in untreated PKU patients found decreased blood concentrations of serotonin, dopamine, and norepinephrine and reduced excretion in the urine of these neurotransmitters and their metabolites such as 5-hydroxyindoleacetic acid (5-HIAA) (26). Reduced levels of neurogenic amines were also found in the $P^{\text {enu2 }}$ mouse $(24,25,27)$. The levels of serotonin, 5-HIAA, dopamine, and norepinephrine were confirmed to be decreased in the brains of the Pahnu2 mice in our colony compared with those in their wild-type and Slc6a19-KO littermates (Figure $3)$. In contrast, we observed significant $(P<0.0001)$ increases in serotonin and 5-HIAA in the brains of the $P_{a h}{ }^{\text {enu2 }} \times$ Slc6a19-KO mice. Moreover, dopamine levels in $P a h^{\text {enu2 }} \times$ Slc6a19-KO mice were comparable to wild-type levels, and the levels of norepinephrine were also increased compared with those in $\mathrm{Pah}^{\text {enu2 }}$ mice (Figure 3, B and D). Thus, KO of Slc6a19 in the $P^{2} h^{\text {enu2 }}$ mouse substantially corrected the deficits in several neurogenic amines critical for cognition and normal brain development.

Reduced astrocyte proliferation in the brains of Pah ${ }^{\text {enu2 }}$ mice lacking SLC6A19. Besides a decrease in neurotransmitter levels, elevated brain Phe has been reported to result in a number of neuropathological alterations in untreated PKU patients and in the $P a h^{\text {enu2 }}$ mouse, including hypomyelination, oxidative stress, and gliosis $(6,28-30)$. We examined different brain regions for evidence of astrocyte proliferation by immunohistochemical staining of brain sections using a glial fibrillary acidic protein antibody. Compared with wild-type animals, we observed in the $P a h^{\text {enu2 }}$ mice an increased number of astrocytes in the ventral pallidum, a structure within the basal ganglia involved in motor functions, motivated behaviors, and working memory $(31,32)$ (Figure 4). The number of astrocytes was at normal levels in Pah ${ }^{\text {enu2 }} \times$ Slc6a19-KO mice, indicating that the reduction of Phe in the brains of $\mathrm{Pah}^{\mathrm{en} 2} \times$ Slc6a19-KO mice was sufficient to ameliorate a critical aspect of the neuropathology associated with PKU.

Increased dendritic spine density and branching in the brains of Pah ${ }^{\text {enu2 }}$ mice lacking SLC6A19. Another adverse consequence of elevated brain Phe is a negative effect on learning and memory. An examination of brains from profoundly retarded, untreated PKU patients found a prominent decrease in synaptic spines of neurons in the prefrontal cortex and other regions and a scarcity of dendritic arborizations $(33,34)$. Given the importance of dendritic spines in learning and memory storage, brains from wild-type, $\mathrm{Pah}^{\text {enu2 }}$, and $\mathrm{Pah}^{\text {enu2 }}$ $\times$ Slc6a19-KO mice were sectioned and subjected to Golgi-Cox staining to visualize neuronal morphology. High-resolution videos were collected and processed to generate 2-dimensional tracings of neurons in the hippocampus and prefrontal cortex, and the number of individual spines along the length of representative 
Table 2. Plasma amino acid levels

\begin{tabular}{|c|c|c|c|c|c|}
\hline Amino acid & $\begin{array}{c}P a h^{\text {enu2 }} \times \text { SIc6a19-KO plasma } \\
(\mu \mathrm{M})\end{array}$ & $\begin{array}{c}P a h^{\text {enu2 }} \times \text { SIc6a19 Het plasma } \\
(\mu \mathrm{M})\end{array}$ & $\begin{array}{c}P_{a h^{\text {enu2 }}} \text { plasma } \\
(\mu \mathrm{M})\end{array}$ & $\begin{array}{l}\text { SIc6a19-KO plasma } \\
(\mu \mathrm{M})\end{array}$ & $\begin{array}{l}\text { Wild-type plasma } \\
(\mu \mathrm{M})\end{array}$ \\
\hline Glycine & $116 \pm 14$ & $134 \pm 19$ & $135 \pm 15$ & $99 \pm 20$ & $160 \pm 18$ \\
\hline Serine & $87 \pm 14$ & $88 \pm 15$ & $94 \pm 13$ & $88 \pm 7$ & $110 \pm 16$ \\
\hline Aspartic acid & $15 \pm 5$ & $12 \pm 6$ & $10 \pm 3$ & $13 \pm 3$ & $11 \pm 4$ \\
\hline Glutamine & $849 \pm 70$ & $690 \pm 148$ & $708 \pm 93$ & $790 \pm 82$ & $788 \pm 82$ \\
\hline Alanine & $245 \pm 44$ & $210 \pm 54$ & $196 \pm 52$ & $222 \pm 33$ & $279 \pm 49$ \\
\hline Cystine & $27 \pm 5$ & $30 \pm 13$ & $31 \pm 12$ & $24 \pm 4$ & $36 \pm 12$ \\
\hline Lysine & $585 \pm 145$ & $388 \pm 69$ & $396 \pm 48$ & $585 \pm 65$ & $464 \pm 85$ \\
\hline Proline & $123 \pm 25$ & $79 \pm 15$ & $79 \pm 15$ & $125 \pm 15$ & $109 \pm 27$ \\
\hline Histidine & $77 \pm 9$ & $91 \pm 19$ & $88 \pm 9$ & $71 \pm 6$ & $90 \pm 11$ \\
\hline Isoleucine & $125 \pm 17$ & $139 \pm 20$ & $129 \pm 15$ & $114 \pm 14$ & $129 \pm 20$ \\
\hline Phenylalanine & $573 \pm 105$ & $1,879 \pm 146$ & $1,875 \pm 328$ & $118 \pm 12$ & $122 \pm 20$ \\
\hline Tryptophan & $69 \pm 14$ & $103 \pm 14$ & $98 \pm 11$ & $61 \pm 10$ & $134 \pm 20$ \\
\hline Valine & $187 \pm 34$ & $204 \pm 37$ & $184 \pm 37$ & $174 \pm 19$ & $197 \pm 33$ \\
\hline
\end{tabular}

neurons was counted. In the CA1 region of the hippocampus, $P a h^{\text {enu2 }}$ mice had a clearly reduced density of dendritic spines compared with wild-type mice (Figure 5A). By comparison, there was no difference in spine density between genotypes in the dentate gyrus. In layers $2 / 3$ (Figure 5A) and layer 5 (data not shown) of the medial prefrontal cortex, $P a h^{\text {enu2 }}$ mice had a significantly $(P<0.005)$ reduced density of spines compared with wild-type mice in both regions. Spine density in Pah ${ }^{\text {enu }} \times$ Slc6a19-KO mice, in contrast, was found to be significantly increased in both the hippocampal CA1 region and layers $2 / 3$ of the medial prefrontal cortex (Figure 5A).

In addition to reduced spine density, tracings from layer $2 / 3$ cortical neurons revealed visually obvious decreased and less complex branching in $\mathrm{Pah}^{\mathrm{en} 2}$ mice compared with wild-type mice (Figure 5, B and C). In contrast, tracings of cortical neurons in $\mathrm{Pah}^{\mathrm{enu} 2} \times$ Slc6a19-KO mice appeared similar to those from wildtype mice (Figure 5D). We quantified dendritic complexity using two different approaches. First, quantification of the total number of branches showed that layer $2 / 3$ cortical neurons have significantly fewer total branches in $P a h^{\text {enu2 }}$ mice $(P<0.0001)$ compared with both wild-type mice and $P a h^{\text {enu2 }} \times S l c 6 a 19-\mathrm{KO}$ mice (Figure $5 \mathrm{E}$ ). Second, a more detailed analysis was performed using a priming scheme, with branches stemming directly from the soma labeled as $1^{\prime}$, branches extending off the $1^{\prime}$ dendrites labeled as $2^{\prime}$, etc. Although there was an equal number of $1^{\prime}$ branches in cortical neurons in $P a h^{\text {enu2 }}$ and wild-type mice, there was a significant reduction in the number of $2^{\prime}-5^{\prime}$ branches in $P a h^{\text {enu2 }}$ mice compared with both wild-type mice and $P a h^{\text {enu2 }} \times$ Slc6a19-KO mice $(P<0.0001)$ (Figure 5F). Thus, while $P a h^{\text {enu2 }}$ mice have abnormal dendritic architecture in critical neurons responsible for learning and memory, Pah ${ }^{\text {enu2 }} \times$ Slc6a19-KO mice exhibit a pronounced increase in both dendritic branch number and branching complexity, comparable to those found in wild-type animals.

Identification of a spatial working memory deficit in Pahenu2 mice and correction of this deficit in Pah ${ }^{\text {enu } 2}$ mice lacking SLC6A19. We next asked if the decrease in plasma and brain Phe levels, the increase in neurotransmitter levels, and the improvements in neuropathology in Pah ${ }^{\text {enu2 }} \times$ Slc6a19-KO mice were sufficient to translate to a meaningful correction of the cognitive deficits of the $\mathrm{Pah}^{\text {enu2 }}$ mouse. A number of behavioral tests performed on the PKU mouse model have identified impairments in various aspects of learning and memory $(22,35)$. Given the evidence of neuropathology in the prefrontal cortex and reported deficits in spatial recognition, we decided to evaluate our mice using an 8-arm radial maze, a sensitive and partially automated test of spatial learning and working memory (36). This "win-shift" test first involved a training 
Table 3. Brain amino acid levels

\begin{tabular}{|c|c|c|c|c|c|}
\hline Amino acid & $\begin{array}{c}P a h^{\text {enu2 }} \times S I c 6 a 19-K 0 \\
\text { brain }(\mu \mathrm{g} / \mathrm{ml})\end{array}$ & $\begin{array}{c}P^{P a h^{\text {enu2 }}} \times \text { Slc6a19 Het } \\
\text { brain }(\mu \mathrm{g} / \mathrm{ml})\end{array}$ & $P_{a h}^{\text {enu2 }}$ brain $(\mu \mathrm{g} / \mathrm{ml})$ & Slc6a19-KO brain $(\mu \mathrm{g} / \mathrm{ml})$ & Wild-type brain $(\mu \mathrm{g} / \mathrm{ml})$ \\
\hline Glycine & $22.7 \pm 7.7$ & $22.1 \pm 2.8$ & $29.3 \pm 9.2$ & $27.1 \pm 7.2$ & $23.4 \pm 6.3$ \\
\hline Serine & $19.9 \pm 0.8$ & $21.8 \pm 2.6$ & $21.9 \pm 1.8$ & $20.9 \pm 3.0$ & $21.8 \pm 4.1$ \\
\hline Asparagine & $3.3 \pm 0.5$ & $3.0 \pm 0.5$ & $2.9 \pm 0.6$ & $3.2 \pm 0.5$ & $3.0 \pm 0.4$ \\
\hline Aspartic acid & $110.9 \pm 11.8$ & $117.6 \pm 29.9$ & $110.9 \pm 19.1$ & $124.9 \pm 22.3$ & $118.7 \pm 22.0$ \\
\hline Glutamine & $203.2 \pm 20.5$ & $151.5 \pm 15.7$ & $157.3 \pm 15.1$ & $224.6 \pm 22.3$ & $205.6 \pm 12.2$ \\
\hline Alanine & $17.3 \pm 6.4$ & $16.9 \pm 7.5$ & $17.6 \pm 8.2$ & $17.5 \pm 6.0$ & $15.8 \pm 5.3$ \\
\hline Cystine & $4.9 \pm 0.3$ & $5.2 \pm 0.6$ & $5.7 \pm 0.6$ & $5.6 \pm 0.4$ & $5.7 \pm 0.3$ \\
\hline Lysine & $9.5 \pm 1.9$ & $8.3 \pm 0.9$ & $8.5 \pm 1.6$ & $9.3 \pm 1.0$ & $8.2 \pm 1.4$ \\
\hline Proline & $2.8 \pm 0.5$ & $2.6 \pm 0.6$ & $2.6 \pm 0.6$ & $2.7 \pm 0.3$ & $2.4 \pm 0.3$ \\
\hline Histidine & $3.0 \pm 1.0$ & $4.2 \pm 0.9$ & $4.7 \pm 1.1$ & $2.8 \pm 0.5$ & $2.8 \pm 0.5$ \\
\hline Isoleucine & $1.4 \pm 0.2$ & $1.2 \pm 0.4$ & $1.2 \pm 0.4$ & $1.6 \pm 0.3$ & $1.6 \pm 0.2$ \\
\hline Phenylalanine & $15.1 \pm 2.2$ & $32.4 \pm 3.3$ & $36.1 \pm 6.1$ & $7.8 \pm 1.1$ & $7.0 \pm 0.6$ \\
\hline Tryptophan & $0.8 \pm 0.1$ & $0.7 \pm 0.1$ & $0.7 \pm 0.1$ & $0.9 \pm 0.2$ & $0.9 \pm 0.1$ \\
\hline Valine & $1.8 \pm 0.2$ & $1.5 \pm 0.3$ & $1.6 \pm 0.3$ & $2.2 \pm 0.2$ & $2.3 \pm 0.2$ \\
\hline
\end{tabular}

phase, where all 8 arms of the maze were open and baited (see Methods), and the ability of the mice to collect the food reward at the end of each arm without revisiting any arm was recorded. All the groups showed improvement during this phase, with no significant difference between the genotypes by days 9-10 (Figure 6, A and B). The result indicates that both $\mathrm{Pah}^{\text {enu2 }}$ and wild-type mice have intact learning acquisition and spatial short-term working memory function. In the study phase, only $4 \mathrm{arms}$ were open and baited. Once the mice completed the study phase, there was a short retention interval of 3 minutes, which was followed by the test phase where the mice were reinserted into the maze, with all 8 arms now open but only the 4 arms previously closed in the study phase being baited. A visit to a food trough previously visited in either the study phase or test phase was recorded as an error. During the study phase at days 1-2 or 9-10, there was no difference between the groups (Figure 6, C and E). However, during the test phase $P_{a h}{ }^{\text {enu2 }}$ mice exhibited a significant increase in the number of errors compared with the other genotypes $(P<0.0001)$, showing no improvement between days 1-2 (Figure 6D) and days 9-10 (Figure 6F). The results indicate that $P a h^{\text {enu2 }}$ mice exhibit a significant deficit in spatial working memory but only after increasing the memory load caused by the delayed spatial win-shift task, which requires the animal to hold spatial information across the retention interval (37). This deficit was fully rescued in Pah ${ }^{\text {enu2 }} \times$ Slc6a19-KO mice (Figure 6F). Thus, the decreased blood and brain Phe levels in Pah ${ }^{\text {enu2 }} \times$ Slc6a19-KO mice translated to a measurable improvement in a specific cognitive deficit.

Efficient knockdown of SLC6A19 expression in the kidney after injection of peptide-linked antisense oligonucleotides. The above results demonstrated the beneficial effects on the PKU disease phenotype in the context of a complete absence of SLC6A19 expression beginning at birth. We wished to determine if transient inhibition of SLC6A19 expression in an adult animal could also correct the disease phenotype. Phosphorodiamidate morpholino oligonucleotides conjugated to cell-penetrating peptides (PPMOs) have been shown to efficiently knock down target mRNA expression in tissues by inhibiting mRNA splicing. We screened oligonucleotides designed to inhibit Slc6a19 splicing in primary mouse renal tubular epithelial cells. The most potent oligonucleotide was conjugated to peptide "J," which has been shown to be particularly effective in the kidney (38). Adult $P a h^{\text {enu2 }}$ mice were injected with 2 consecutive doses of either SLC6A19 PPMO (19e6ei-J) or scrambled control PPMO (Scrmbl-J) on days 0 and 1 and then injected once weekly thereafter for 3 weeks. This dosing regimen was well 
A
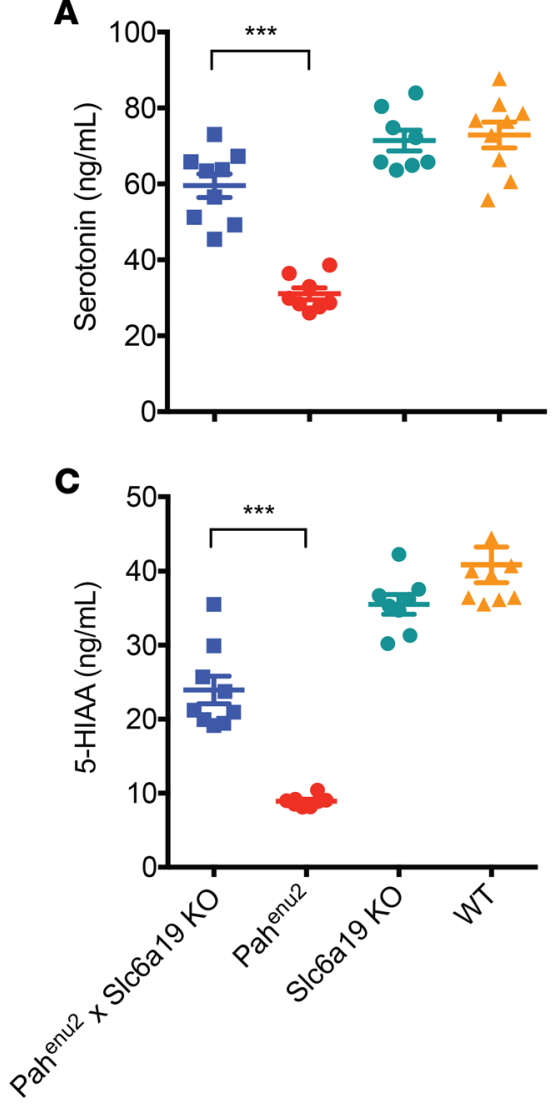

B

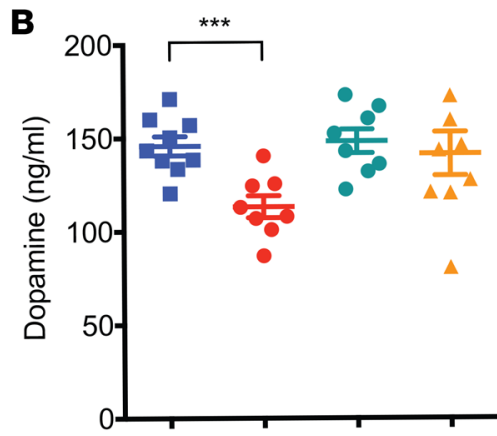

D

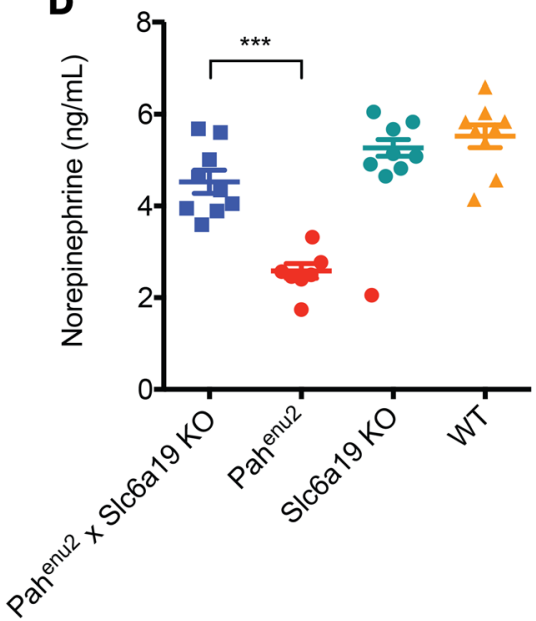

Figure 3. Increased levels of neurogenic amines in the brains of Pah ${ }^{\text {enu2 }}$ mice lacking SIc6A19. (A) Serotonin, (B) dopamine, (C) 5-hydroxyindoleacetic acid (5-HIAA), and (D) norepinephrine. Animals were perfused with PBS and whole brains were harvested. Neurotransmitters were quantitated by HPLC/ tandem MS/MS. $n=8-10$ animals/group. One-way ANOVA was performed, follow by Tukey's multiple comparisons test. ${ }^{* *} P<0.0001$. Mean \pm SEM is shown.

tolerated and found to provide the optimal knock down of SLC6A19 but did induce transient mild tubular necrosis and regeneration (Supplemental Figure 1; supplemental material available online with this article; https://doi.org/10.1172/jci.insight.121762DS1). Kidneys were harvested 3 days after the last dose. There was essentially complete knockdown of SLC6A19 mRNA and protein expression in 19e6ei-J PPMO-treated animals (Figure 7, A and B). Immunohistochemical staining of kidney sections showed abundant expression of SLC6A19 in the proximal tubule epithelial cells of control animals (Figure 7C) but no visible SLC6A19 expression in 19e6ei-J PPMO-treated mice. Thus, PPMO treatment was highly effective in inhibiting SLC6A19 expression in the $P a h^{\text {enu2 }}$ mouse.

Extensive and sustained efficacy of oligonucleotide-mediated inhibition of SLC6A19 in Pahenu2 mice. We next measured the levels of Phe in the urine, blood, and brains of mice treated with the Slc6a19 or control PPMO. After 24 hours of urine collection, barely detectable amounts of Phe were found in the urine of wild-type animals as expected, and only small quantities of Phe were present in the urine of animals treated with either saline or the control PPMO (Figure 8A). However, abundant amounts of Phe ( $\sim 20 \mathrm{mg}$ ) were excreted over a 24-hour period from mice treated with the Slc6a19 PPMO, indicating successful induction of neutral aminoaciduria. Plasma Phe levels in the animals treated with the control PPMO or saline were in the typical 2,000 $\mu \mathrm{M}$ range (Figure $8 \mathrm{~B}$ ). In contrast, plasma Phe levels in mice treated with the Slc6a19 PPMO were decreased by approximately $50 \%$ (Figure $8 \mathrm{~B}$ ), and these levels remained remarkably constant at $840-870 \mu \mathrm{M}$ throughout the course of the study. These levels were higher than what was observed in Pahenu2 $\times$ Slc6a19-KO animals $(\sim 600 \mu \mathrm{M})$, which was not unexpected given the complete absence of SLC6A19 expression in all tissues in the KO animals versus inhibition of only kidney SLC6A19 with the PPMOs. Brain Phe levels in the Slc6a19 PPMO-treated mice decreased approximately $45 \%$ relative to the control PPMO- or saline-treated animals (Figure $8 \mathrm{C}$ ), with levels only slightly higher than those found in wild-type mice. Thus, antisense oligonucleotide treatment was effective in inducing neutral aminoaciduria that in turn significantly lowered blood and brain Phe levels.

Given the critical importance of neurogenic amines on cognitive function, it was of interest whether Slc6a19 PPMO treatment could completely or partially restore the deficiency of serotonin, dopamine, 


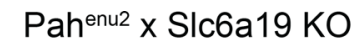

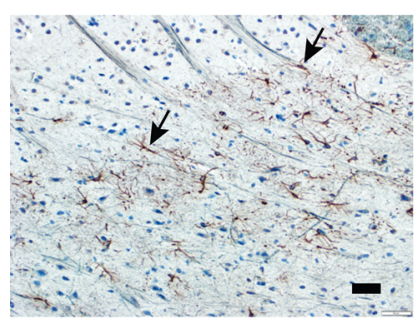

Slc6a19 KO

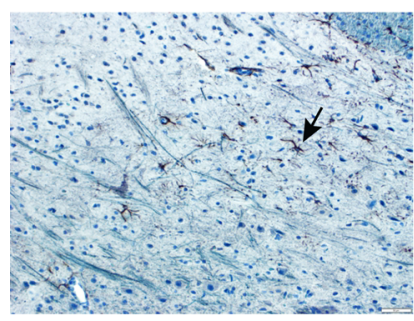

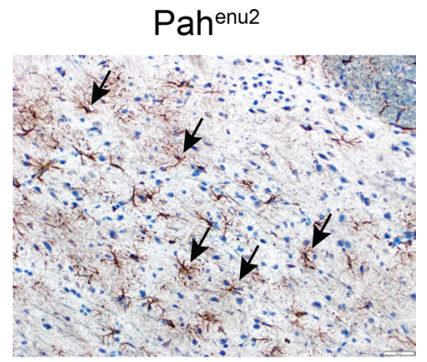

WT

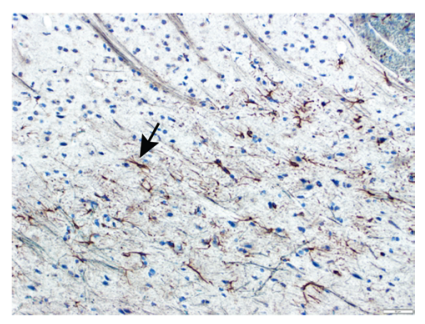

Figure 4. Decreased number of reactive astrocytes in the ventral pallidum region of Pah ${ }^{\text {enu2 }}$ mice lacking SIc6a19 compared with $\boldsymbol{P a h}^{\text {enu2 }}$ mice. Brains from the different genotypes of mice were sectioned and stained with an anti-GFAP antibody, and the number of immunoreactive astrocytes was counted. Arrows indicate representative dark brown-stained, starshaped astrocytes. Scale bar: $50 \mu \mathrm{m}$. $n$ = 5 animals/group. One-way ANOVA was performed, follow by Tukey's multiple comparisons test. ${ }^{* *} P=0.003$. Mean \pm SEM is shown.

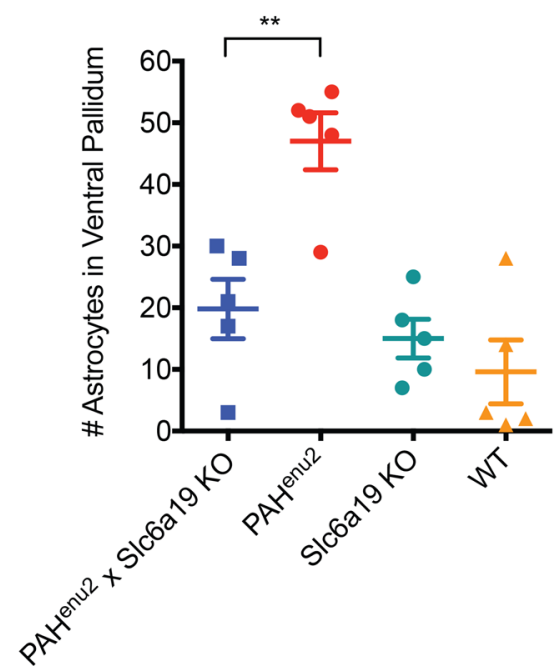

and norepinephrine in the Pah ${ }^{\text {enu2 }}$ mouse. Twenty-five days after the initiation of Slc6a19 PPMO treatment, the levels of brain serotonin and 5-HIAA increased significantly compared with those of animals treated with the control PPMO or saline (Figure 8, D and F). In addition, dopamine and norepinephrine increased to levels comparable to those in wild-type animals (Figure 8, E and G). The results demonstrate that deficits in neurogenic amines in the PKU mouse could be substantially restored by antisense-mediated inhibition of SLC6A19 in an adult animal.

\section{Discussion}

Intracellular and circulating Phe concentrations that are normally maintained at fairly constant levels are disrupted in PKU, due to impairment in the initial step of Phe metabolism. While one therapeutic approach is to restore the catabolic pathway, in this study we investigated an alternate idea of removing excess Phe by preventing its reabsorption by the kidney. Amino acids are normally very efficiently reabsorbed from the glomerular filtrate by transporters located on the apical membrane of epithelial proximal tubule cells, with SLC6A19 being the major transporter for neutral amino acids such as Phe. KO mice lacking SLCA19 exhibit aminoaciduria but are otherwise phenotypically normal. To ascertain the Phe-lowering potential of targeting SLC6A19, the Pah ${ }^{\text {enu2 }}$ mouse was crossed with the Slc6a19-KO mouse. These $P a h^{\text {enu2 }} \times$ Slc6a19-KO animals excreted abundant amounts of Phe in their urine and were found to have significantly lower blood and brain Phe levels. Thus, blocking SLC6A19 function provided an alternate path for Phe disposal, markedly lowering its accumulation in blood and tissues. 
A

CA1

WT
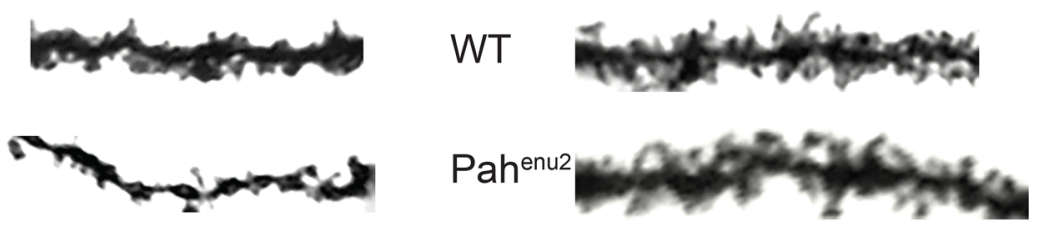

mPFC L2/3

Dentate Gyrus

WT

WT

Pahenu2

Pahenu2
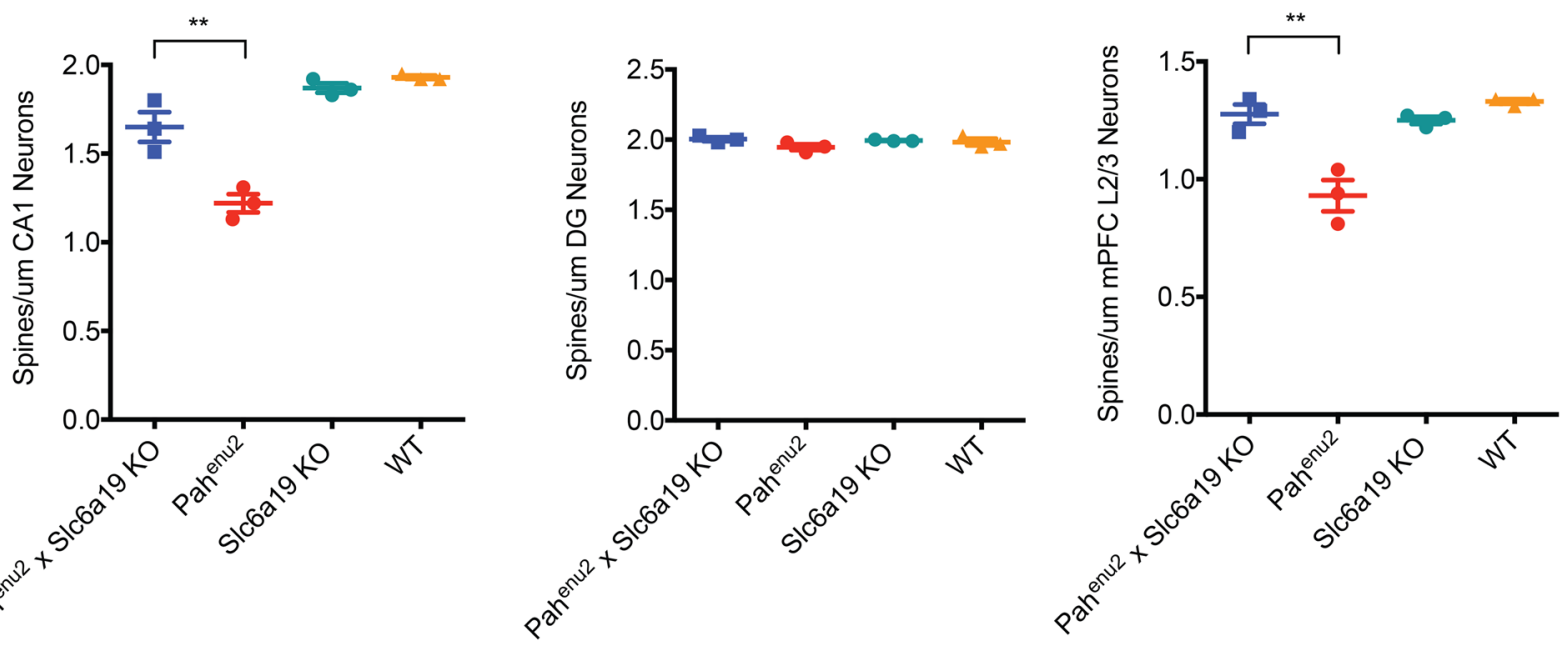

B

C

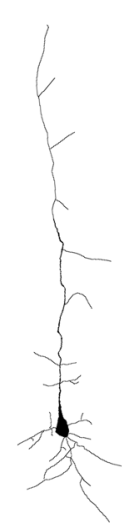

D

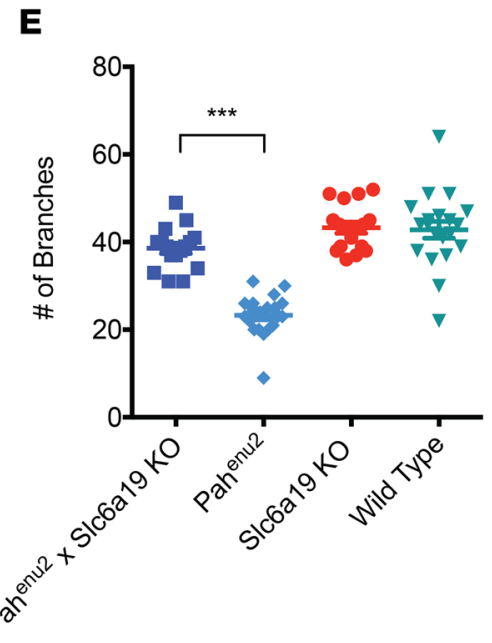

$\mathbf{F}$

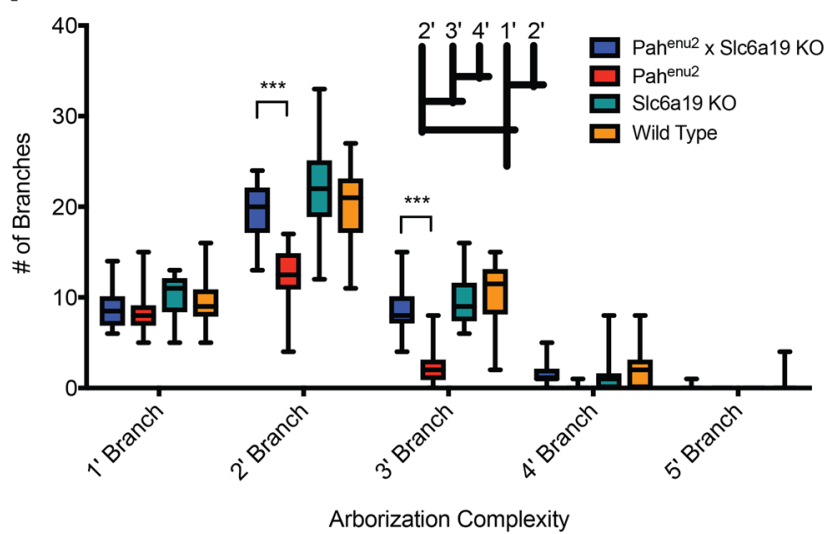


Figure 5. Increased dendritic spine density and arborization complexity in Pahur mice lacking SIc6a19. (A) Dendritic spines were visualized using Golgi-Cox staining. Shown are representative images from the CA1 and dentate gyrus regions of the hippocampus and layers $2 / 3$ of the medial prefrontal cortex. A minimum of 750 spines from 5 neurons per mouse and at least 3 mice per genotype were analyzed. Representative neurons from Pah ${ }^{\text {enu2 }}$, wild-type, and $P a h^{\text {enu2 }} \times$ SIc6a19-KO mice from layers 2/3 of the cortex were visualized using Golgi-Cox staining and electronically traced using Neurolucida Suite and Cell Sens software. Original magnification, $\times 1,000$. (B) Pah $h^{\text {enu2 }} \times$ Slc6a19-KO neuron. (C) Pah ${ }^{\text {enu2 }}$ neuron. (D) Wild-type neuron. (E) Quantification of branch number. $n=16-20$ neurons analyzed per genotype. (F) Quantification of branch complexity. Branch complexity was quantified using a priming scheme, with branches directly connected to the neuronal cell body identified as $1^{\prime}$, secondary branch points identified as $2^{\prime}$, etc. One-way ANOVA was performed, follow by Tukey's multiple comparisons test. ${ }^{* *} P<0.005,{ }^{* *} P<0.001$. Mean \pm SEM is shown.

Interestingly, although blood Phe levels decreased dramatically, the levels of the other circulating amino acids were not significantly altered in $P a h^{\text {enu2 }}$ mice lacking SLC6A19. The reason for this is not fully understood. Reduced muscle protein turnover and a shift in metabolism away from utilization of amino acids as a source of energy may play a role (39). Amino acid transporters may act in concert with mTOR to maintain plasma amino acid homeostasis (40). There may also be increased absorption of small peptides in the small intestine.

In this regard, since SLC6A19 plays an essential role in absorption of Phe in the small intestine, one question is the relative importance of inhibiting intestinal amino acid uptake versus renal amino acid reabsorption. The antisense data indicate that Phe reduction can be achieved in this model by selectively targeting SLC6A19 in the kidney, since intravenous injection of PPMOs had no effect on the expression of SLC6A19 in the small intestine. The amount of Phe reduction was somewhat greater in the $\mathrm{Pah}^{\text {enu2 }}$ mice lacking SLC6A19 compared with that in antisense oligonucleotide-treated animals, suggesting that the absence of Phe uptake via intestinal SLC6A19 may have contributed a minor role to the Phe lowering. On the other hand, we have observed that genetic deletion of TMEM27 in Pah ${ }^{\text {enu2 }}$ mice yielded nearly identical results with regard to urine, blood, and brain Phe levels as deletion of SLC6A19 (Supplemental Figure 2). $P a h^{\text {enu2 }} \times$ TMEM27 KO mice lack expression of SLC6A19 in the kidney but retain expression of SLC6A19 in the small intestine, since intestinal SLC6A19 partners with ACE2 instead of TMEM27. Thus, while $P a h^{\text {enu2 }} \times$ Slc6a19-KO mice are unable to uptake Phe via SLC6A19 in the small intestine, whereas $P a h^{\text {enu2 }}$ $\times$ TMEM27 KO mice presumably have normal Phe uptake, both mutant/KO animals exhibited nearly identical amounts of Phe reduction (Supplemental Figure 2). Altogether, these findings support further investigation into blockade of renal reabsorption of Phe as a potential therapeutic modality.

However, another consideration is that the data indicate that blockade of SLC6A19 has to be nearly complete in order to induce the degree of urinary aminoaciduria needed to lower blood Phe levels. This level of inhibition was achieved with the Slc6a19 PPMOs (Figure 7), but toxicity issues known to be associated with PPMOs need to be addressed prior to their utilization beyond proof-of-concept studies (41) (Supplemental Figure 1). Fortunately, highly specific and potent small-molecule inhibitors have been developed against several members of the SLC6A family of transporters, e.g., the widely prescribed selective serotonin reuptake inhibitors, suggesting that SLC6A19 may be pharmacologically amenable to targeting.

Mice lacking SLC6A19 exhibit no overt phenotype other than chronic aminoaciduria, and most, but not all, humans lacking SLC6A19 also exhibit no obvious symptoms. Hartnup disorder is an autosomal recessive disease that is caused by mutations in the gene encoding SLC6A19 (42, 43). With an incidence of approximately 1:30,000, Hartnup disorder is one of the most frequent disorders of amino acid metabolism after PKU $(44,45)$. Clinical features associated with Hartnup disorder include a pellagra-like photosensitive skin rash, cerebellar ataxia, and psychiatric disturbances (44). Many Hartnup patients have increased excretion of indoles, as indolic compounds are formed in the gut from bacteria that consume and degrade tryptophan that fails to be absorbed by the small intestine due to the lack of SLC6A19. The decrease in tryptophan absorption results in decreased niacin synthesis, which can be readily treated with nicotinamide. However, the vast majority of patients with Hartnup disorder are asymptomatic and do not require treatment (46). Why some Hartnup patients develop symptoms and others do not is still unclear, but, in many cases, the underlying factor in patients that do develop symptoms appears to be poor nutrition or a very low-protein diet (47). Overall, Hartnup disorder can be considered benign, given the few reported cases of Hartnup relative to the number of subjects identified by newborn screening (44).

To what extent the mouse model data described herein may be applicable to humans is unknown, but there are important species differences to be considered. First, while the Pah ${ }^{\text {enu2 }}$ mouse mimics the severe, classic PKU phenotype, mice have a much higher metabolic rate and, thus, intake greater than 20-40 times 
Training Phase

8 Doors open

8 Arms baited
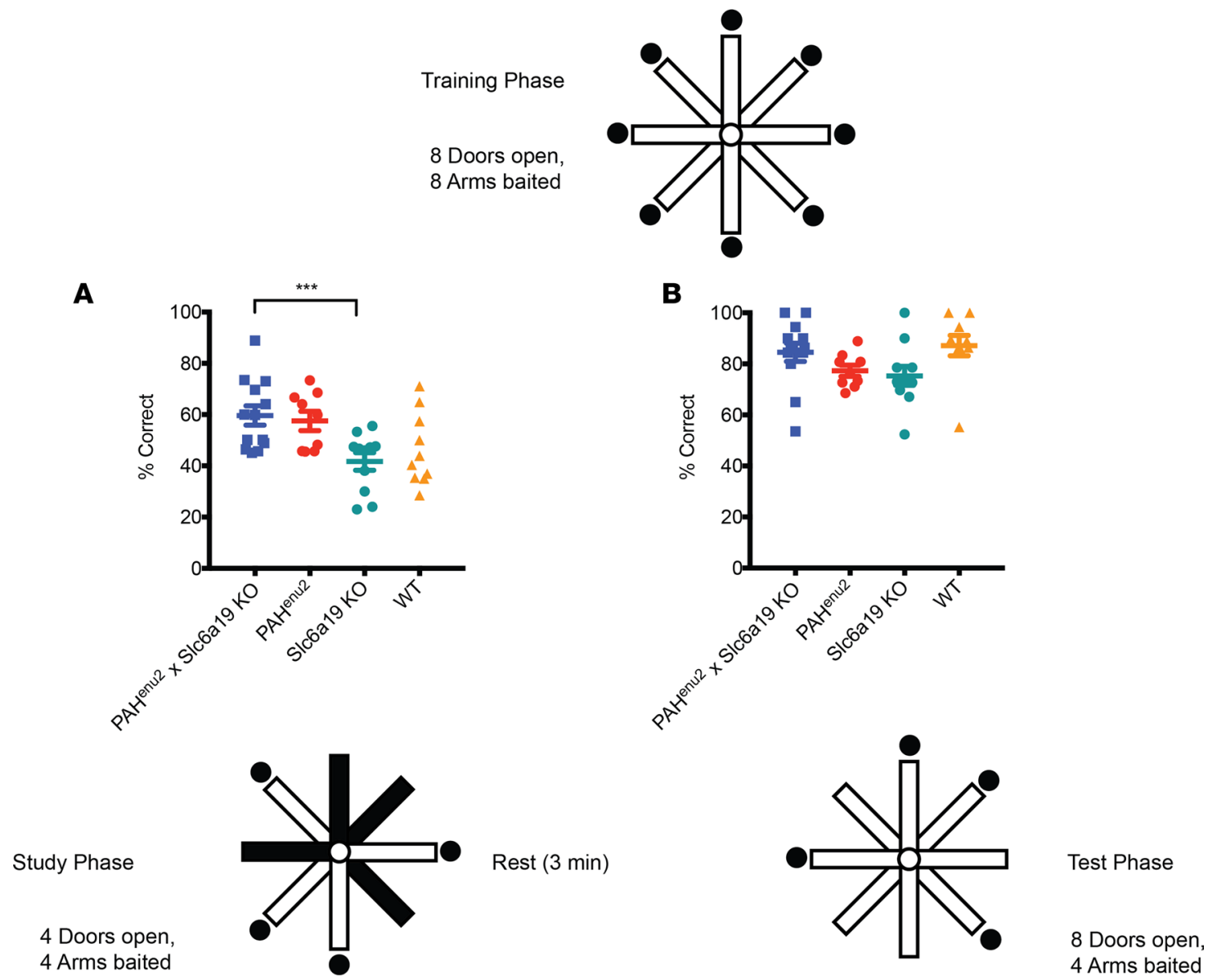

4 Doors open,

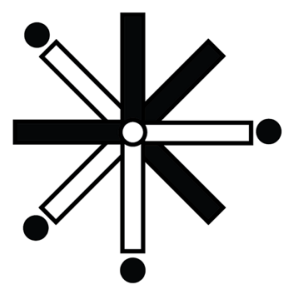

4 Arms baited

C

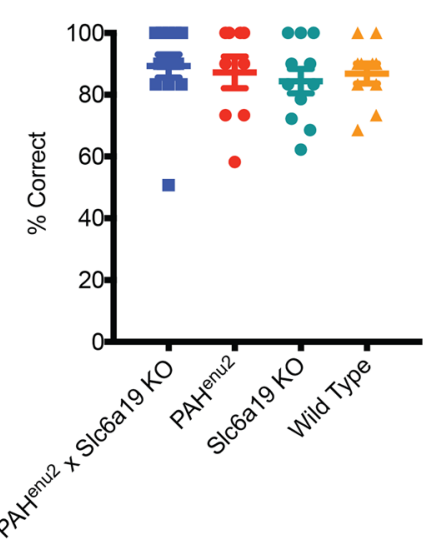

E

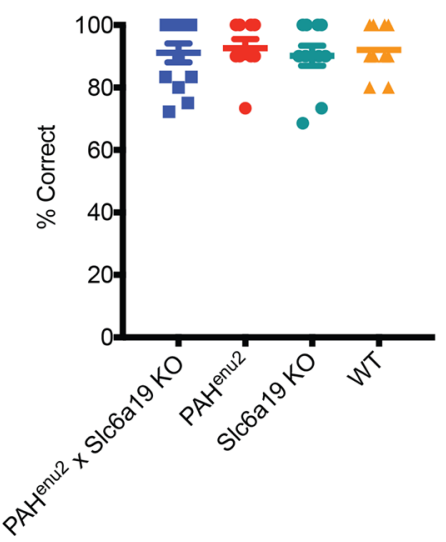

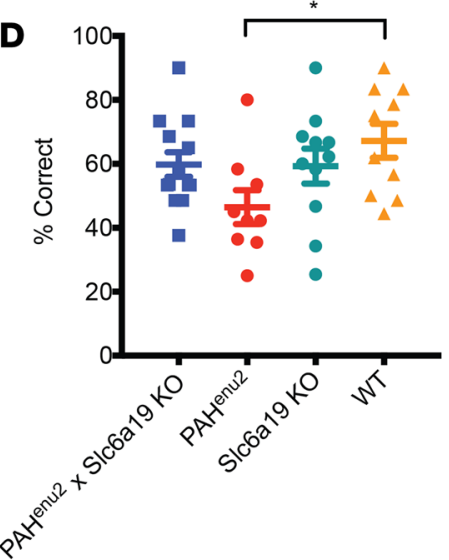

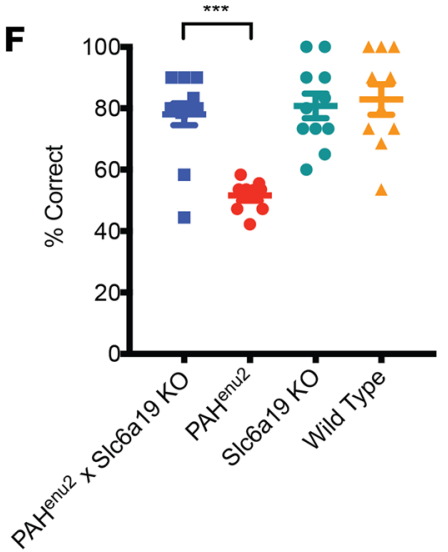


Figure 6. Characterization of the deficit in spatial working memory in Pahur mice and restoration in Pahu2 mice lacking SIc6a19. Mice were tested in an 8-arm radial arm maze (see Methods). Each black circle indicates food reward at the end of an arm; white bars indicate arms open to entry; black bars indicate arms blocked from entry. (A) Percentage of correct choices made during the training phase, days 1-2. (B) Training phase days, 9-10. (C) Study phase, days 11-12. (D) Test phase, days 11-12. (E) Study phase, days 19-20. (F) Test phase, days 19-20. $n=9-13$ animals/group. One-way ANOVA was performed, follow by Tukey's multiple comparisons test. ${ }^{*} P=0.0375,{ }^{* *} P<0.0001$. Mean \pm SEM is shown.

the amount of Phe per kilogram of body weight compared with humans. Second, while the decrease in plasma Phe levels in mice lacking SLC6A19 was quite substantial, the levels ( $600 \mu \mathrm{M}$ for the KO animals and $\sim 900 \mu \mathrm{M}$ for the Slc6a19 PPMO-treated mice) were well above the normal range of $60-120 \mu \mathrm{M}$. Third, while mice lacking SLC6A19 were smaller at weaning compared with their wild-type counterparts (Figure 1), the effect of inhibiting SLC6A19 on growth in young children, perhaps in the context of a Phe-restricted diet, is unknown and will need to be carefully assessed.

Finally, PKU is one member of a group of diseases where normal amino acid metabolism is impaired. Examples include intoxication-type disorders caused by defects in enzymes involved in the catabolism of branched-chain amino acids, such as maple syrup urine disease, where accumulation of leucine and its metabolites causes neurotoxicity and the risk of life-threatening metabolic decompensation (48). Another member is type II tyrosinemia, where a deficiency in the enzyme involved in the first step of tyrosine catabolism leads to toxic accumulation of tyrosine, causing corneal and dermatological lesions as well as neurocognitive deficits in some patients (49). Similar complications may arise from the use of nitisinone to treat type I tyrosinemia, which greatly improves clinical outcomes but also elevates tyrosine levels (50). In addition, there are diseases that cause a secondary accumulation of amino acids, e.g., urea cycle disorders (UCDs). With UCDs, in the absence of a functioning urea cycle the body attempts to rid excess ammonia via incorporation of nitrogen into glutamine, alanine, and other amino acids, but those amino acids are efficiently reabsorbed by the kidney. Moreover, excess brain glutamine itself is believed to be toxic (51). The potential positive or negative effects of inhibiting neutral amino acid reabsorption in these diseases are currently being investigated.

\section{Methods}

Generation of transgenic mice. BTBR-Pah $h^{\text {enu2 } / J ~ m i c e ~(s t o c k ~ 002232) ~ w e r e ~ p u r c h a s e d ~ f r o m ~ T h e ~ J a c k s o n ~ L a b-~}$ oratory. Slc6a19-KO mice were obtained from the Taconic Knockout Repository (Taconic). The mouse was originally created by targeted insertion of a selection cassette into the Slc6a19 gene by Lexicon Pharmaceuticals. The background strain of the mouse is 129/SvEv-C57BL/6. Heterozygous Pah $^{\text {enu2 }}$ females were mated with heterozygous Slc6a19-KO males to obtain offspring heterozygous for both alleles. These mice were then intercrossed to create homozygous Pah ${ }^{\text {enu2 }} \times$ Slc6a19-KO mice. Animals were cared for in an AAALAC-accredited facility in accordance with the guidelines established by the National Research Council. Animals had access to food and water ad libitum.

Amino acid analysis. Quantitation of amino acids was performed by UPLC-MS/MS, using an Acquity UPLC (Waters Corporation) hyphenated to an API 5000 triple quadrupole mass spectrometer (AB SCIEX). Samples were prepared as follows: $10 \mu$ plasma was combined with $110 \mu 1$ mobile phase A $(0.5 \%$ trifluoroacetic acid, $0.3 \%$ heptafluorobutyric acid in water $)$ and $20 \mu 1$ methanol. The sample was vortexed and centrifuged (9,300 $\mathrm{g}$ for 5 minutes). A $50-\mu 1$ aliquot of the supernatant was further diluted with mobile phase A to a final dilution factor of $200 \times$ and $4,000 \times$. Both dilutions were analyzed. Standard curves were prepared in the $200 \times$ and $4,000 \times$ diluted matrix. $50 \mu 1$ urine was combined with $450 \mu 1$ of $0.1 \mathrm{~N} \mathrm{HCl}$. A $10-\mu 1$ aliquot of the diluted urine was further diluted with mobile phase A to a final dilution factor of $1,000 \times$ and $10,000 \times$. Both dilutions were analyzed. Standard curves were prepared in mobile phase A. Brains were weighed and homogenized in ( $1 \mathrm{mM}$ oxalic acid, 3 mM L-cysteine, 0.1 M acetic acid) (52) using TissueLyser II (Qiagen). A 20- $\mu$ l aliquot of homogenate was combined with $20 \mu 1$ mobile phase A and $20 \mu 1$ methanol. The sample was vortexed and then centrifuged. An aliquot of the supernatant was diluted with mobile phase A to a final dilution factor of $300 \times$ and $3,000 \times$. Both dilutions were analyzed. Standard curves were prepared in the $300 \times$ and $3,000 \times$ diluted matrix.

L-amino acids purchased from MilliporeSigma were used to prepare standard solutions. Amino acid analysis was performed using a Waters Acquity BEH C18 column $(1.7 \mu \mathrm{m}, 2.1 \mathrm{~mm} \times 100 \mathrm{~mm})$ (Waters Corporation) with gradient separation, which included a 0.5 -minute hold at $99.9 \%$ mobile phase A $(0.5 \%$ trifluoroacetic acid, 
A

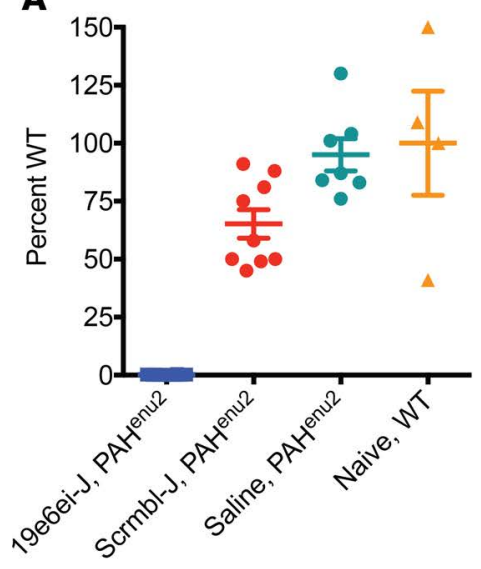

C
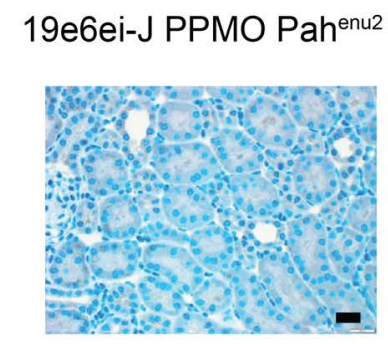

Saline Pahenu2

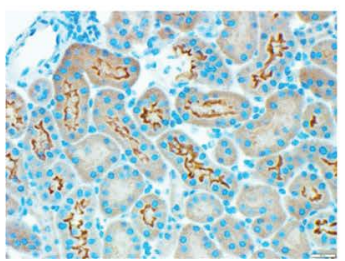

B

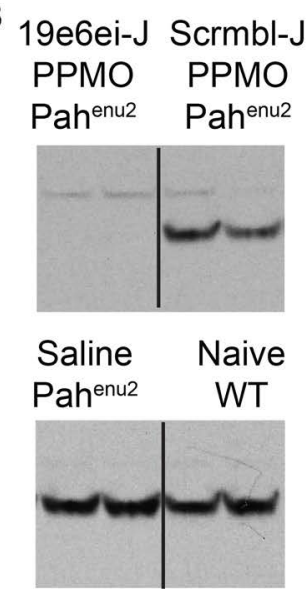

Scrmbl-J PPMO Pahenu2

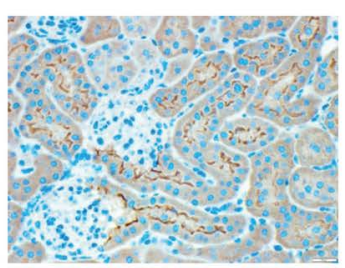

Naive WT

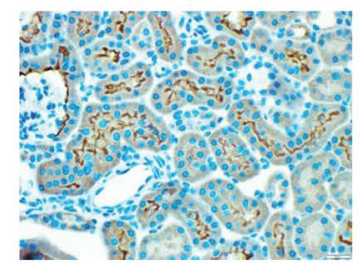

Figure 7. Oligonucleotide-mediated inhibition of SLC6A19 in Pah $\boldsymbol{h}^{\text {enu2 }}$ mice. (A) Knockdown of SLC6A19 RNA and protein in the kidneys of PPMO-treated mice. Animals were injected intravenously $(7.5 \mathrm{mg} / \mathrm{kg})$ with SIc6a19 PPMO (19e6ei-J), control PPMO (Scrmbl-J), or saline on days 0,1 , 8,15 , and 22 . Kidneys were harvested on day 25. SLC6A19 mRNA levels were measured by quantitative RT-PCR. (B) SLC6A19 protein levels in the kidney were visualized by Western blotting. (C) Immunohistochemical staining of kidney sections using an anti-SLC6A19 antibody. Slides were counterstained with hematoxylin and eosin. Original magnification, $\times 400$. Scale bar: 100 $\mu \mathrm{m} . n=10$ animals/PPMO-treated groups; $n=8$ animals/saline-treated group, saline; $n=4$ animals/naive wildtype group. Mean \pm SEM is shown

$0.3 \%$ heptafluorobutyric acid in water) followed by a $0.1 \%-30 \%$ mobile phase B (acetonitrile) gradient over 2.5 minutes, an increase to $90 \%$ B over 3 minutes, a 1-minute wash at $90 \% \mathrm{~B}$, and reequilibration at $99.9 \% \mathrm{~A}$ for 1.9 minutes. Total run time was 6.5 minutes, injection volume was $5 \mu$, flow rate was $0.5 \mathrm{ml} / \mathrm{min}$, column temperature was $25^{\circ} \mathrm{C}$, and the sample temperature was $10^{\circ} \mathrm{C}$. MS/MS detection was carried out in positive ion mode with declustering potential and collision energy manually optimized to 20 and 25 , respectively, while the ion-spray potential and temperature were set to $1,500 \mathrm{~V}$ and $500^{\circ} \mathrm{C}$.

Measurement of brain neurotransmitters. Mice were perfused with PBS and then brains were dissected, snap frozen in liquid nitrogen, and stored at $-80^{\circ} \mathrm{C}$. Brains were homogenized at a concentration of 200 $\mathrm{mg} / \mathrm{ml}$ in $1 \mathrm{mM}$ oxalic acid, $3 \mathrm{mM}$ cysteine, $0.1 \mathrm{M}$ acetic acid, $\mathrm{pH} \sim 3.6$, using a TissueLyser II (Qiagen), centrifuged at $15,000 \mathrm{~g}$ for 10 minutes at $4^{\circ} \mathrm{C}$, and the supernatant was transferred to a new tube. A 100$\mu \mathrm{l}$ aliquot of the supernatant was diluted with $100 \mu \mathrm{l}$ of $600 \mu \mathrm{g} / \mathrm{ml}$ cysteine buffer and then $80 \mu 1$ of the $2 \times$ diluted sample was evaporated under nitrogen and reconstituted in $40 \mu \mathrm{l}$ of a solution containing $90 \%$ mobile phase A ( $0.25 \%$ trifluoroacetic acid, $0.15 \%$ heptafluorobutyric acid in $300 \mathrm{ng} / \mathrm{ml}$ cysteine) and $10 \%$ mobile phase B (acetonitrile). Standards for dopamine, serotonin, 5-hydroxyindole-3-acetic acid, and norepinephrine were purchased from MilliporeSigma. Standard curves were prepared in the diluent solution.

Analysis of neurotransmitters was performed by UPLC-MS/MS using an Acquity UPLC (Waters Corporation) hyphenated to an API 5000 triple-pole quadrupole spectrometer (AB SCIEX). A Phenomenex Luna C18-HST column $(3 \times 100 \mathrm{~mm}, 2.5 \mu \mathrm{M})$ was used with gradient separation, which included a 0.5 -minute hold at $90 \%$ A followed by a $10 \%-25 \%$ B gradient over 2.5 minutes, washing at $95 \% \mathrm{~B}$, and reequilibration at $90 \% \mathrm{~A}$. Total run time was 6.5 minutes, injection volume was $5 \mu 1$, column temperature was $25^{\circ} \mathrm{C}$, and the sample temperature was $5^{\circ} \mathrm{C}$. Flow rate was $0.5 \mathrm{ml} / \mathrm{min}$. MS/MS detection was carried out in positive ion mode with declustering potential and collision energy manually optimized for each analyte, while the ion-spray potential and temperature were set to $5,000 \mathrm{~V}$ and $500^{\circ} \mathrm{C}$. 
A

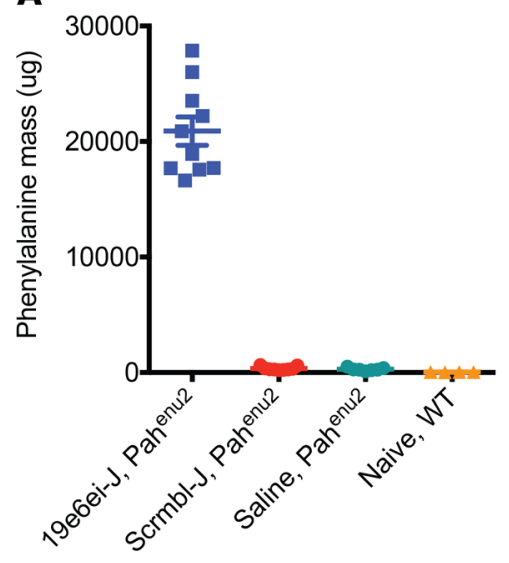

B

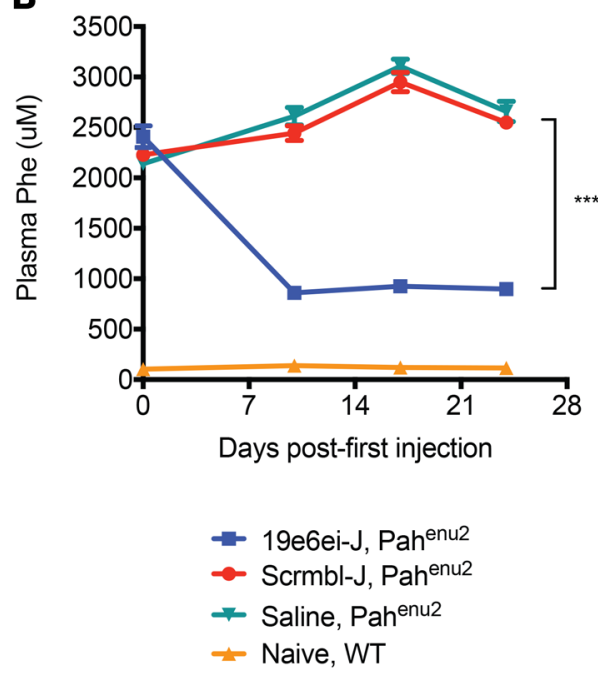

C

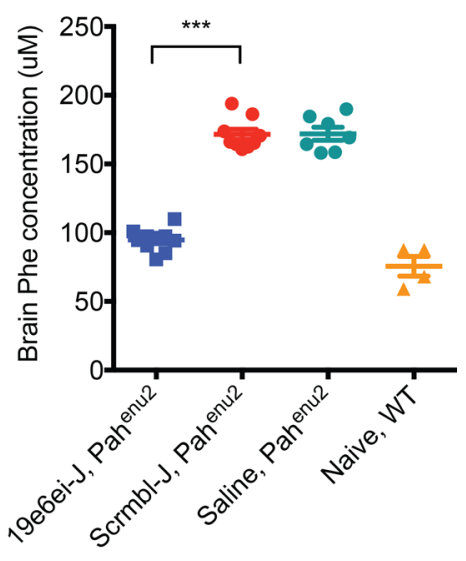

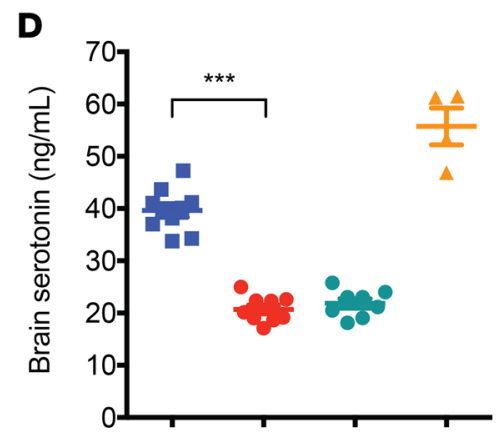
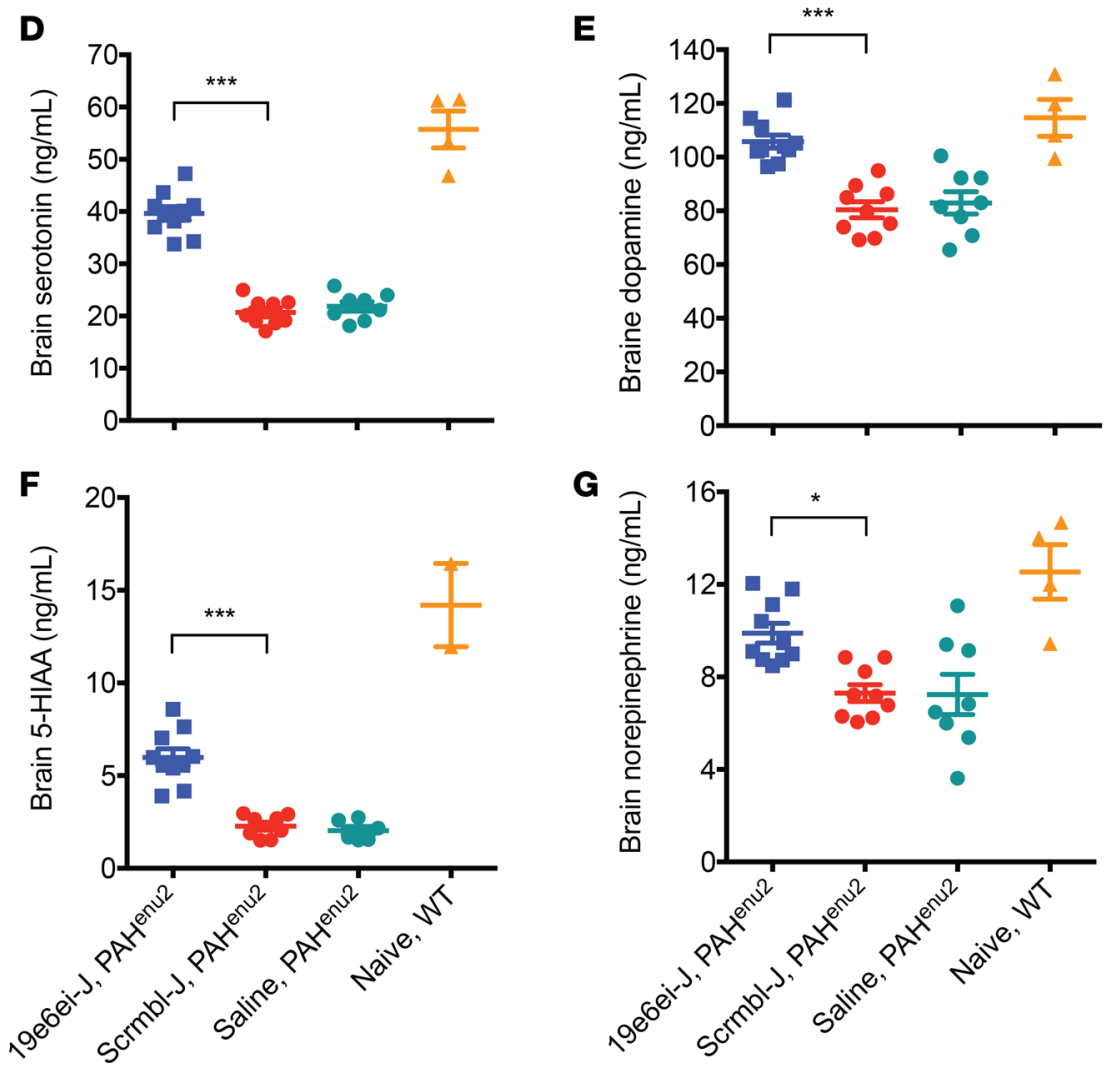

Figure 8. Efficacy following oligonucleotide-mediated inhibition of SLC6A19 in Pahu2 mice. Animals were injected intravenously (7.5 mg/kg) with SIc6a19 PPMO (19e6ei-J), control PPMO (Scrmbl-J), or saline on days $0,1,8,15$, and 22. PPMO treatment induced (A) excretion of Phe in urine and (B) significantly lowered plasma and (C) brain Phe levels in Pahenu2 mice. Urine was collected for 24 hours on days $24-25$. Blood was collected on days 10,18 , and 25 . Animals were perfused with PBS on day 25 , and whole brains were harvested. Phenylalanine was quantitated by HPLC/tandem MS/ MS. (D-G) PPMO treatment increased brain neurotransmitter levels in Pahenuz mice. (D) Brain serotonin, (E) dopamine, (F) 5-HIAA, and (G) norepinephrine levels were quantitated by high-performance liquid chromatography/tandem mass spectrometry. $n=10$ animals/PPMO-treated groups; $n=8$ animals/saline-treated group; $n=4$ animals/naive wild-type group. One-way ANOVA was performed, follow by Tukey's multiple comparisons test. ${ }^{*} P=0.0178,{ }^{* * *} P<0.0001$. Mean \pm SEM is shown. 
Synthesis, purification, and administration of peptide-linked morpholino oligonucleotides to mice. Several phosphorodiamidate morpholino oligonucleotides (PMOs) were designed and synthesized by GeneTools LLC to inhibit splicing of mouse Slc6a19. The PMOs were screened by transfection of primary mouse renal tubular epithelial cells using the Endo-Porter peptide delivery agent (GeneTools LLC) and quantitative RT-PCR. The most potent oligo, 19e6-ei (exon 6-intron 6 splice donor) had the following sequence: 5'-CССТTCACCTCCATACTCACTGCAC-3'. The scrambled control oligo had the following sequence: 5'-ATCACCATTCCGCCTACCTTCCACC-3'.

The PMOs were synthesized with a 5' primary amine modification and functionalized by reaction with sulfo-N-succinimidyl-4-formylbenzoate to generate PMOs with a 5' 4-formylbenzamido modification. A cell-penetrating peptide based on peptide "J" (38) was synthesized with an additional C-terminal lysine functionalized by 6-hydrazino-nicotinamide ( $\mathrm{HyNic}$ ) modification on the epsilon carbon. The sequence of peptide $\mathrm{J}$ is as follows: Ac-rXrrXrrXrrXrXBK(HyNic)-NH2, where Ac indicates N-terminal acetyl; NH2 indicates $\mathrm{C}$-terminal amide; $\mathrm{r}$ indicates $\mathrm{D}$-arginine; $\mathrm{X}$ indicates 6-amino-hexanoic acid; $\mathrm{B}$ indicates $\beta$-alanine; and $\mathrm{K}(\mathrm{HyNic})$ indicates L-lysine-e-HyNic.

Functionalized PMO and peptide were reacted to form peptide-PMO conjugates containing a bis-arylhydrazone linkage. PPMO conjugates were purified from unconjugated peptide and PMO by reverse-phase HPLC. Briefly, samples were reconstituted in buffer A (20 mM ammonium acetate) and loaded on an XBridge preparative C18 column (Waters Corporation) at $5 \mathrm{ml} / \mathrm{min}$. One-minute fractions were collected with a gradient of $2 \%-95 \%$ buffer B (20 mM ammonium acetate, $60 \%$ acetonitrile) over 48 minutes. Fractions containing both 265 and $354 \mathrm{~nm}$ absorbance were pooled and lyophilized, reconstituted in deionized $\mathrm{H}_{2} \mathrm{O}$, and quantified by A265 and A354. Aliquots of purified PPMO were lyophilized and reconstituted in PBS prior to injection.

Mice were injected intravenously at a dose of $7.5 \mathrm{mg} / \mathrm{kg}$ for 2 consecutive days and then once weekly thereafter. Mice were bled via the mandibular vein 3 days after the third, fourth, and fifth injection of PPMO. Mice were placed in metabolic cages on day 24 for 24-hour urine collection.

Immunohistochemistry. Animals were perfused with PBS, and dissected brains and kidneys were fixed in $10 \%$ neutral buffered formalin in PBS, $\mathrm{pH} 7.4$, at $4^{\circ} \mathrm{C}$ for 2 days; dehydrated in a graded series of $50 \%, 75 \%$, $90 \%, 96 \%$, and $100 \%$ ethanol; equilibrated with xylene, embedded in paraffin; and sectioned at a thickness of $8 \mu \mathrm{m}$. Mounted paraffin sections were dewaxed in xylene and rehydrated in graded ethanol solutions prior to antigen retrieval in boiling $50 \mathrm{mM}$ sodium citrate plus $0.01 \%$ Tween 20 for 25 minutes. Endogenous peroxidase activity was inhibited through incubation of sections in $3 \%$ hydrogen peroxide for 10 minutes prior to washing with PBS and blocking with $10 \mathrm{mg} / \mathrm{ml}$ BSA in PBS for 2 hours at room temperature. Sections were incubated with rabbit anti-GFAP (1:5,000) (Abcam) or rabbit anti-SLC6A19 (1:500) (custom-made genomic antibody, Sdix) primary antibodies overnight at $4^{\circ} \mathrm{C}$. Secondary biotinylated goat anti-rabbit or rabbit anti-rat antibodies were used at 1:200 dilution for 1 hour at room temperature. Sections were then incubated with streptavidin-HRP polymer complex (1:1,000) (Vector Laboratory) for 30 minutes. Slices were washed 3 times for 5 minutes each between antibodies and enzyme incubations with PBS containing $0.4 \%$ Tween-20. Diaminobenzidine (DAB) enhanced substrate system was used according to the manufacturer's instructions (Cell Signaling Technology). After washing off excess DAB substrate, slides were counterstained with Gill's No. 2 Hematoxylin (MilliporeSigma) prior to coverslip mounting. Sections were viewed with an Olympus BX43 microscope equipped with a DP-73 camera.

Dendritic spine density and arborization. Brains were removed from mice, and cryosections were cut at thickness of $100 \mu \mathrm{M}$ and collected onto gelatin-coated slides (FD NeuroTechnologies Inc.). After air drying, Golgi-Cox staining was performed using the FD Rapid GolgiStain Kit (FD NeuroTechnologies Inc.) according to the manufacturer's instructions. Slides were viewed with an Olympus BX43 microscope equipped with MPLAPON objectives and a DP-73 camera (Olympus America). Neurolucida Suite (MBF Bioscience) and CellSens (Olympus America) software were used to generate and analyze neuron traces from layer II/III cortical neurons.

Behavioral test. Learning and memory assessments were conducted using an 8-arm radial mouse maze (Med Associates). This maze consists of 8 arms extending from a central chamber with 8 guillotine doors positioned at the interface of the central chamber and arms. A 14-mg food dispenser and trough are at the end of each arm. Each arm has 2 sets of photosensors to track movement of mice in and out of the arms. In addition, the food trough also contains photosensors that detect mouse head entries into food troughs and to dispense food. The sides and top of each arm are composed of clear plastic to allow mice to use visual cues in the room to spatially navigate the maze. A computer in an adjacent room controlled 
the maze events and data collection. Photosensor, food, and door data were collected using MED-PC software v4.39 (Med Associates) with a resolution of $10 \mathrm{~ms}$.

Thirteen days prior to the start of behavioral testing, body weights from individually housed mice were taken over an average of 3 days. Mice were diet restricted to reduce and maintain a body weight of no less than $87.0 \%$ of their ad libitum food body weight for the duration of the behavioral assessment. For 4 days prior to testing, mice were pretrained to associate the maze with the experience of obtaining a 14-mg sucrose-flavored food reward (Bio-Serve, F05684) by allowing each animal free access to 4 of the 8 arms until one food reward from each arm was retrieved. The maze was cleaned between subjects with Clidox at a ratio of 1:18:1 (Pharmacal Research Laboratories Inc.) to prevent a previous mouse's scent from interfering with a subsequent mouse's performance. To further prevent a mouse from using its own scent cues, the entire maze was scent saturated using cotton bedding from the mouse's home cage prior to mice entering the maze.

Radial arm maze training phase (8 arms open, 8 arms baited) to assess spatial short-term memory. Approximately 20-week-old mice were individually placed in the central chamber of the maze for a 1-minute acclimation period before beginning the procedure. After acclimation, all 8 doors opened with the objective of collecting a food reward available at the end of each arm ( 8 arms open, 8 arms baited). Only one food reward is delivered per arm, and a revisit to a previously visited food trough is considered an error in spatial short-term memory. After either collecting the last food reward or after 15 minutes of elapsed time, the session ends and the doors close. These training phase sessions are performed once a day, at the same time of day for each mouse for 10 consecutive days. Results are reported as the mean total error \pm SEM from the first 2 days or last 2 days of training. Following 10 days of consecutive spatial short-term memory training, animals proceeded directly to the spatial working memory test with a retention interval.

Radial arm maze test phase (delayed spatial win shift) to assess spatial working memory. Mice were tested using a delayed spatial win-shift task for 10 consecutive days. This is a 2-phase procedure composed of a study phase and test phase. In the study phase, animals were placed in the central chamber of the maze for a 1-minute acclimation period. Four of the eight doors were opened (randomly chosen by the computer every day, but the same for all mice each day), and the mouse was required to collect a food reward that was available at the end of each of the 4 baited arms ( 4 arms open, 4 arms baited). After collecting the last food reward (or 15 minutes of elapsed time), the doors closed. The mouse was subjected to a retention interval (time delay) by being taken out of the maze and returned to his home cage for 3 minutes, during which the maze was cleaned. The mouse was returned to the maze to begin the test phase. After 1 minute of acclimation, all 8 doors of the maze open. Only arms that were previously closed in the study phase were baited in the test phase ( 8 arms open, 4 arms baited). A revisit to a food trough previously visited in either phase is considered an error in spatial working memory. After either collecting the last food reward or after 15 minutes of elapsed time, the session ends and the doors close. Results are reported as the mean total error \pm SEM obtained from the first 2 days or last 2 days of the test phase.

Statistics. Data were analyzed by 1-way ANOVA followed by Tukey's post-test using Prism 7 software (GraphPad Software). $P<0.05$ was considered statistically significant.

Study approval. All animal studies were reviewed and approved by Sanofi's Institutional Animal Use and Care Committee.

\section{Author contributions}

AMB identified the target; MP, EG, MF, AMB, and NSY performed the experiments; MF conducted the neuropathology and behavioral studies; SG and AK conducted the mass spectrometry and analysis; SHC and YZ provided project guidance; and NSY designed the experiments and wrote the manuscript.

\section{Acknowledgments}

We thank the In Vivo Research Center staff for assistance with the animal studies and Takako Moriguchi and Peter Hallock for critically reading the manuscript.

Address correspondence to: Nelson S. Yew or Adam M. Belanger, Sanofi, 49 New York Avenue, Framingham, Massachusetts 01701, USA. Phone: 508.270.2414; Email: nelson.yew@sanofi.com (N.S. Yew). Phone: 508.270.2479; adam.belanger@sanofi.com (A.M. Belanger).

MF's present address is: Shire, Lexington, Massachusetts, USA. 
1. Scriver CR, Kaufman S. In: Scriver CR, et al, eds. The Metabolic and Molecular Bases of Inherited Disease. New York: McGraw-Hill; 2001:1667-1724.

2. Williams RA, Mamotte CD, Burnett JR. Phenylketonuria: an inborn error of phenylalanine metabolism. Clin Biochem Rev. 2008;29(1):31-41.

3. Donlon J, Sarkissian C, Levy H, Scriver CR. In: Beaudet AL, et al, eds. The Online Metabolic and Molecular Bases of Inherited Disease. New York, NY: The McGraw-Hill Companies, Inc.; 2014. https://ommbid.mhmedical.com/content.aspx?bookid=971\&sectionid=62673211. Accessed July 20, 2018.

4. Blau N. Genetics of phenylketonuria: then and now. Hum Mutat. 2016;37(6):508-515.

5. Blau N, Thöny B, Cotton RGH, Hyland K. In: Beaudet AL, et al, eds. The Online Metabolic and Molecular Bases of Inherited Disease. New York, NY: The McGraw-Hill Companies, Inc.; 2014. https://ommbid.mhmedical.com/content.aspx?bookid=971\&sectionid=62673465. Accessed July 20, 2018.

6. Anderson PJ, Leuzzi V. White matter pathology in phenylketonuria. Mol Genet Metab. 2010;99 Suppl 1:S3-S9.

7. Smith CB, Kang J. Cerebral protein synthesis in a genetic mouse model of phenylketonuria. Proc Natl Acad Sci USA. 2000;97(20):11014-11019.

8. Qin M, Smith CB. Regionally selective decreases in cerebral glucose metabolism in a mouse model of phenylketonuria. J Inherit Metab Dis. 2007;30(3):318-325.

9. Walter JH, White FJ. Blood phenylalanine control in adolescents with phenylketonuria. Int J Adolesc Med Health. 2004;16(1):41-45.

10. Camp KM, et al. Phenylketonuria Scientific Review Conference: state of the science and future research needs. Mol Genet Metab. 2014;112(2):87-122.

11. Levy HL, Ghavami M. Maternal phenylketonuria: a metabolic teratogen. Teratology. 1996;53(3):176-184

12. Waisbren SE, et al. Maternal phenylketonuria: long-term outcomes in offspring and post-pregnancy maternal characteristics. JIMD Rep. 2015;21:23-33.

13. Makrides V, Camargo SM, Verrey F. Transport of amino acids in the kidney. Compr Physiol. 2014;4(1):367-403.

14. Bröer S. Amino acid transport across mammalian intestinal and renal epithelia. Physiol Rev. 2008;88(1):249-286.

15. Bröer A, Klingel K, Kowalczuk S, Rasko JE, Cavanaugh J, Bröer S. Molecular cloning of mouse amino acid transport system B0, a neutral amino acid transporter related to Hartnup disorder. J Biol Chem. 2004;279(23):24467-24476.

16. Danilczyk U, et al. Essential role for collectrin in renal amino acid transport. Nature. 2006;444(7122):1088-1091.

17. Malakauskas SM, et al. Aminoaciduria and altered renal expression of luminal amino acid transporters in mice lacking novel gene collectrin. Am J Physiol Renal Physiol. 2007;292(2):F533-F544.

18. Fairweather SJ, et al. Molecular basis for the interaction of the mammalian amino acid transporters B0AT1 and B0AT3 with their ancillary protein collectrin. J Biol Chem. 2015;290(40):24308-24325.

19. Camargo SM, et al. Tissue-specific amino acid transporter partners ACE2 and collectrin differentially interact with hartnup mutations. Gastroenterology. 2009;136(3):872-882.

20. Bröer A, et al. Impaired nutrient signaling and body weight control in a Na+ neutral amino acid cotransporter (Slc6a19)-deficient mouse. J Biol Chem. 2011;286(30):26638-26651.

21. Shedlovsky A, McDonald JD, Symula D, Dove WF. Mouse models of human phenylketonuria. Genetics. 1993;134(4):1205-1210.

22. Cabib S, Pascucci T, Ventura R, Romano V, Puglisi-Allegra S. The behavioral profile of severe mental retardation in a genetic mouse model of phenylketonuria. Behav Genet. 2003;33(3):301-310.

23. Zagreda L, Goodman J, Druin DP, McDonald D, Diamond A. Cognitive deficits in a genetic mouse model of the most common biochemical cause of human mental retardation. J Neurosci. 1999;19(14):6175-6182.

24. Pascucci T, Ventura R, Puglisi-Allegra S, Cabib S. Deficits in brain serotonin synthesis in a genetic mouse model of phenylketonuria. Neuroreport. 2002;13(18):2561-2564.

25. Puglisi-Allegra S, Cabib S, Pascucci T, Ventura R, Cali F, Romano V. Dramatic brain aminergic deficit in a genetic mouse model of phenylketonuria. Neuroreport. 2000;11(6):1361-1364.

26. Güttler F, Lou H. Dietary problems of phenylketonuria: effect on CNS transmitters and their possible role in behaviour and neuropsychological function. J Inherit Metab Dis. 1986;9 Supp1 2:169-177.

27. Pascucci T, et al. In vivo catecholaminergic metabolism in the medial prefrontal cortex of ENU 2 mice: an investigation of the cortical dopamine deficit in phenylketonuria. J Inherit Metab Dis. 2012;35(6):1001-1009.

28. Rocha JC, Martins MJ. Oxidative stress in phenylketonuria: future directions. J Inherit Metab Dis. 2012;35(3):381-398.

29. Schuck PF, Malgarin F, Cararo JH, Cardoso F, Streck EL, Ferreira GC. Phenylketonuria Pathophysiology: on the Role of Metabolic Alterations. Aging Dis. 2015;6(5):390-399.

30. Vargas CR, Wajner M, Sitta A. Oxidative stress in phenylketonuric patients. Mol Genet Metab. 2011;104 Suppl:S97-S99.

31. Root DH, Melendez RI, Zaborszky L, Napier TC. The ventral pallidum: Subregion-specific functional anatomy and roles in motivated behaviors. Prog Neurobiol. 2015;130:29-70.

32. Smith KS, Tindell AJ, Aldridge JW, Berridge KC. Ventral pallidum roles in reward and motivation. Behav Brain Res. 2009;196(2):155-167.

33. Bauman ML, Kemper TL. Morphologic and histoanatomic observations of the brain in untreated human phenylketonuria. Acta Neuropathol. 1982;58(1):55-63.

34. Kornguth S, Gilbert-Barness E, Langer E, Hegstrand L. Golgi-Kopsch silver study of the brain of a patient with untreated phenylketonuria, seizures, and cortical blindness. Am J Med Genet. 1992;44(4):443-448.

35. Andolina D, et al. 5-Hydroxytryptophan during critical postnatal period improves cognitive performances and promotes dendritic spine maturation in genetic mouse model of phenylketonuria. Int J Neuropsychopharmacol. 2011;14(4):479-489.

36. Babb SJ, Crystal JD. Episodic-like memory in the rat. Curr Biol. 2006;16(13):1317-1321.

37. Floresco SB, Seamans JK, Phillips AG. Selective roles for hippocampal, prefrontal cortical, and ventral striatal circuits in radial-arm maze tasks with or without a delay. J Neurosci. 1997;17(5):1880-1890.

38. Jearawiriyapaisarn N, et al. Sustained dystrophin expression induced by peptide-conjugated morpholino oligomers in the muscles of mdx mice. Mol Ther. 2008;16(9):1624-1629.

39. Jiang Y, et al. Mice lacking neutral amino acid transporter B(0)AT1 (Slc6a19) have elevated levels of FGF21 and GLP-1 and 
improved glycaemic control. Mol Metab. 2015;4(5):406-417.

40. Taylor PM. Role of amino acid transporters in amino acid sensing. Am J Clin Nutr. 2014;99(1):223S-230S.

41. Moulton HM, Moulton JD. Morpholinos and their peptide conjugates: therapeutic promise and challenge for Duchenne muscular dystrophy. Biochim Biophys Acta. 2010;1798(12):2296-2303.

42. Seow HF, et al. Hartnup disorder is caused by mutations in the gene encoding the neutral amino acid transporter SLC6A19. Nat Genet. 2004;36(9):1003-1007.

43. Kleta R, et al. Mutations in SLC6A19, encoding B0AT1, cause Hartnup disorder. Nat Genet. 2004;36(9):999-1002.

44. Levy HL. In: Beaudet AL, et al, G eds. The Online Metabolic and Molecular Bases of Inherited Disease. New York, NY: The McGraw-Hill Companies, Inc.; 2014. https://ommbid.mhmedical.com/content.aspx?bookid=971\&sectionid=62654267. Accessed July 20, 2018.

45. Wilcken B, Smith A, Brown DA. Urine screening for aminoacidopathies: is it beneficial? Results of a long-term follow-up of cases detected bny screening one millon babies. J Pediatr. 1980;97(3):492-497.

46. Wilcken B, Yu JS, Brown DA. Natural history of Hartnup disease. Arch Dis Child. 1977;52(1):38-40.

47. Scriver CR, et al. The Hartnup phenotype: Mendelian transport disorder, multifactorial disease. Am J Hum Genet. 1987;40(5):401-412.

48. Knerr I, Weinhold N, Vockley J, Gibson KM. Advances and challenges in the treatment of branched-chain amino/keto acid metabolic defects. J Inherit Metab Dis. 2012;35(1):29-40.

49. Mitchell GA, Grompe M, Lambert M, Tanguay RM. In: Beaudet AL, et al, eds. The Online Metabolic and Molecular Bases of Inherited Disease. New York, NY: The McGraw-Hill Companies, Inc.; 2014. https://ommbid.mhmedical.com/content.aspx?bookid=971\&sectionid=62673883. Accessed July 20, 2018.

50. Mitchell GA, Yang H. Remaining challenges in the treatment of tyrosinemia from the clinician's viewpoint. Adv Exp Med Biol. 2017;959:205-213.

51. Albrecht J, Norenberg MD. Glutamine: a Trojan horse in ammonia neurotoxicity. Hepatology. 2006;44(4):788-794.

52. Kankaanpää A, Meririnne E, Ariniemi K, Seppälä T. Oxalic acid stabilizes dopamine, serotonin, and their metabolites in automated liquid chromatography with electrochemical detection. J Chromatogr B Biomed Sci Appl. 2001;753(2):413-419. 